\title{
Article \\ Semidefinite Multiobjective Mathematical Programming Problems with Vanishing Constraints Using Convexificators
}

\author{
Kin Keung Lai ${ }^{1, *, \dagger}$, Mohd Hassan ${ }^{2, \dagger}$, Sanjeev Kumar Singh ${ }^{2, \dagger}$, Jitendra Kumar Maurya ${ }^{3, \dagger}$ and \\ Shashi Kant Mishra ${ }^{2,+}$ \\ 1 International Business School, Shaanxi Normal University, Xi'an 710119, China \\ 2 Department of Mathematics, Institute of Science, Banaras Hindu University, Varanasi 221005, India; \\ mohd.hassan10@bhu.ac.in (M.H.); sanjeevk.singh1@bhu.ac.in (S.K.S.); shashikant.mishra@bhu.ac.in (S.K.M.) \\ 3 Kashi Naresh Government Postgraduate College, Gyanpur, Bhadohi 221304, India; \\ jitendrak.maurya1@bhu.ac.in \\ * Correspondence: mskklai@outlook.com \\ + These authors contributed equally to this work.
}

check for

updates

Citation: Lai, K.K.; Hassan, M.;

Singh, S.K.; Maurya, J.K.; Mishra, S.K Semidefinite Multiobjective Mathematical Programming Problems with Vanishing Constraints Using Convexificators. Fractal Fract. 2022, 6, 3. https://doi.org/10.3390/ fractalfract6010003

Academic Editor: Savin Treanţă

Received: 17 November 2021 Accepted: 19 December 2021 Published: 22 December 2021

Publisher's Note: MDPI stays neutral with regard to jurisdictional claims in published maps and institutional affiliations.

Copyright: (C) 2021 by the authors Licensee MDPI, Basel, Switzerland. This article is an open access article distributed under the terms and conditions of the Creative Commons Attribution (CC BY) license (https:// creativecommons.org/licenses/by/ $4.0 /)$.

\begin{abstract}
In this paper, we establish Fritz John stationary conditions for nonsmooth, nonlinear, semidefinite, multiobjective programs with vanishing constraints in terms of convexificator and introduce generalized Cottle type and generalized Guignard type constraints qualification to achieve strong $S$-stationary conditions from Fritz John stationary conditions. Further, we establish strong $S$-stationary necessary and sufficient conditions, independently from Fritz John conditions. The optimality results for multiobjective semidefinite optimization problem in this paper is related to two recent articles by Treanta in 2021. Treanta in 2021 discussed duality theorems for special class of quasiinvex multiobjective optimization problems for interval-valued components. The study in our article can also be seen and extended for the interval-valued optimization motivated by Treanta (2021). Some examples are provided to validate our established results.
\end{abstract}

Keywords: multiobjective programs with vanishing constraints; semidefinite programming; convexificators; nonsmooth analysis; constraint qualifications

\section{Introduction}

Nonlinear semidefinite programming problems $(S D P)$ include several classes of optimization problems, such as linear programming, quadratic programming, second order cone programming [1], and semidefinite programming [2]. The nonlinear semidefinite programming problem has broad applications in system control [3], truss topology optimization [4], and other several fields. It has been at the center point of optimization research for the last two decades. For instance, in the release of library COMPleib [5], where 168 test examples on nonlinear semidefinite programs from various fields, such as control system design, academia, and many real-life based problems are collected.

In this paper, we consider the following semidefinite multiobjective mathematical programs with vanishing constraints $(S-M M P V C)$,

$$
\begin{aligned}
\min \mathfrak{f}(A) & =\left(\mathfrak{f}_{i}(A), \ldots, \mathfrak{f}_{p}(A)\right) \\
\text { subject to } A \in M & =\left\{A \in \mathbb{M}_{+}^{n}: \mathscr{H}_{i}(A) \geqq 0, \mathscr{G}_{i}(A) \mathscr{H}_{i}(A) \leqq 0\right\},
\end{aligned}
$$

where $M_{+}^{n}$ is set of $n \times n$ positive semidefinite matrix, $\mathfrak{f}_{i}: \mathbb{M}_{+}^{n} \rightarrow \mathbb{R} \cup\{+\infty\}(i=1, \ldots p)$ and $\mathscr{G}_{i}, \mathscr{H}_{i}: \mathbb{M}_{+}^{n} \rightarrow \mathbb{R} \cup\{+\infty\}(i=1, \ldots, m)$ are extended real-valued locally Lipschitz functions.

Nonlinear semidefinite programming problems consist of the nonlinear problems where vector variables are replaced by symmetric positive semidefinite matrices. Nonlinear SDPs have been studied extensively due to a wide range of applications, see for 
instance, [6,7]. Shapiro [6] established first and second order necessary and sufficient optimality conditions under the convexity assumptions. Forsgren [8] extended those results for nonconvex semidefinite programming. Further, Sun et al. [7] and Sun [9] discussed the algorithmic approaches to solve nonlinear semidefinite programming problems. Yamashita and Yabe [10] introduced some numerical methods to solve nonlinear SDP and studied the algorithmic consequences. Recently, Golestani and Nobakhtian [11] proposed the generalized Abadie constraint qualification (GACQ) and established necessary and sufficient optimality conditions for nonlinear semidefinite programming problems using convexificators.

Mathematical programs with vanishing constraints $(M P V C)$ has many applications in truss topology optimization [12], pathfinding problem with logic communication constraints in robot motion planning [13], mixed integer nonlinear optimal control problems [14], scheduling problems with disjoint feasible regions in power generation dispatch [15] and many more fields of the current research [16-18]. Initially, mathematical programs with vanishing constraints (MPVC) was introduced by Achtziger and Kanzow in 2008. MPVC is closely related to an optimization problem known as mathematical programs with equilibrium constraints (MPEC), for more details on MPEC, we refer, [19-28].

Due to the constraints $\mathscr{G}_{i}(z) \mathscr{H}_{i}(z) \leq 0$, the feasible set may not be convex even disconnected, most of the basic constraint qualifications such as linearly independent constraint qualification and Mangasarian-Fromovitz constraint qualification do not hold, therefore, standard Karush-Kuhn-Tucker conditions are of no use in such cases. Several constraint qualifications and necessary optimality conditions have been established in [12] for mathematical programs with vanishing constraints. First order sufficient optimality conditions, as well as second order necessary and sufficient optimality conditions, have been discussed in [29] using generalized convexity for mathematical programs with vanishing constraints. In [30], various stationary conditions under weaker assumptions of constraint qualifications were derived. Further, Hoheisel and Kanzow [31] investigated necessary and sufficient optimality conditions through Abadie and Guignard type constraint qualifications for mathematical programs with vanishing constraints. For more details on the MPVC, we refer to $[16,32,33]$ and the references therein.

Multiobjective optimization problems (MOP) plays a vital role in science, technology, business, economics, and many others field of daily demand, where optimal decisions need to be taken among many conflicting objectives and all objective functions to be optimized simultaneously. Effect of conflict on objectives leads to some change in the solution of (MOP) compared to the optimal solution of single-objective optimization problems. Therefore, weak efficient point (weak Pareto optimal solution), efficient point (Pareto optimal solution) like terms are coined for the solutions of (MOP). Initially, the concept of Pareto optimal solutions was given by Italian civil engineer and economist Vilfredo Pareto and was applied in the studies of economic efficiency and income distribution. Basic concept and literature on the solution of multiobjective optimization problems can be found [34,35]. Maeda [36] studied the strong KKT optimality conditions and differentiable functions. Preda and Chitescu [37] extends these results for semidifferentiable functions. Further, $\mathrm{Li}$ [38] discussed these results for the nonsmooth case. Recently, Lai et al. [39] proposed saddle point necessary and sufficient Pareto optimality conditions for multiobjective convex optimization problems. Treanta [40] established dual pair of multiobjective interval-valued variational control problems. Further, Treanta [41] discussed duality theorems for special class of quasiinvex multiobjective optimization problems for interval-valued components.

Since nonsmoothness in optimization is naturally generated from the mathematical formulation of real-world problems, therefore, proper effective way for solving these problems should be discovered. Even the solution of some smooth problems, sometimes requires the use of nonsmooth optimization techniques, in order to either make it easy or simplify its form. Thus, the field of nonsmooth optimization is an important branch of mathematical programming that is based on classical concepts of variational analysis and generalized derivatives. In recent years, research in nonsmooth analysis has focused on the growth of generalized subdifferentials that give sharp results and good calculus 
rules for nonsmooth functions. It is convexificators [42], that has been used to extend, unify, and sharpen the results in various aspects of optimization. Jeyakumar and Luc [43] provided a more sophisticated version of convexificators by introducing the new notion of convexificators which are the closed set but not necessarily bounded or convex. The new version of convexificators consists only finitely many points so it is advantageous for application point of view. We have used the convexificator due to Jeyakumar and Luc [43] in our study.

Recently, Dorsch et al. [44] established a new result for nonlinear semidefinite programming (NLSDP) where almost all linear perturbations of a given NLSDP are shown to be nondegenerate. Semidefinite programming is a powerful framework from convex optimization that has striking potential for data science applications [45]. Sequential optimality conditions have played a vital role in unifying and extending global convergence results for several classes of algorithms for general nonlinear optimization, Andreani et al. [46] extended these concepts for nonlinear semidefinite programming. Andreani et al. [47] discussed simple extensions of constant rank-type constraint qualifications to semidefinite programming, which are based on the Approximate Karush-Kuhn-Tucker necessary optimality condition and on the application of the reduction approach.

Motivated by the above mentioned work, we propose some new constraints qualification to establish necessary and sufficient type optimality conditions for nonsmooth, nonlinear, semidefinite, multiobjective mathematical programs with vanishing constraints. The organization of this article is as follows: In Section 2, we recall some needful preliminaries and fundamental results. In Section 3, we establish Fritz John necessary optimality conditions and propose generalized Cottle and generalized Guignard type constraint qualification to establish strong Karush-Kuhn-Tucker necessary optimality conditions. Further, sufficient optimality conditions are also established under generalized convexity. Section 4, presents the conclusion of the paper, as well as some possible views towards future work.

\section{Preliminaries}

This section recalls needful notation, definitions, and preliminaries that will be used throughout the paper. $\mathbb{M}^{n}$ is denoted as the space of $n \times n$ symmetric matrices. The notation $A \succeq 0(A \succ 0)$ means that $A$ is a positive semidefinite matrix (positive definite matrix) and we denote by $\mathbb{M}_{+}^{n}\left(\mathbb{M}_{++}^{n}\right)$ the set of all positive semidefinite matrices (positive definite matrices). The inner product of the symmetric matrices $P, Q \in \mathbb{M}^{n}$ is denoted by $\langle P, Q\rangle$ and defined by $\langle P, Q\rangle=\operatorname{tr}(P Q)$ where $\operatorname{tr}($.$) denotes the summation of the diagonal$ elements of a square matrix. The inner product of $x=\left(x_{1}, \ldots, x_{n}\right), y=\left(y_{1}, \ldots, y_{n}\right) \in \mathbb{R}^{n}$ is denoted and defined by $x^{T} y=\sum_{i=1}^{n} x_{i} y_{i}$. The norm associated with matrix inner product is called the Frobenius norm $\|P\|_{F}=\operatorname{tr}(P P)^{\frac{1}{2}}=\left(\sum_{i, j=1}^{n} a_{i j}^{2}\right)^{\frac{1}{2}}$. The vector space $\mathbb{M}^{n}$ with this norm is a Hilbert space and $\mathbb{M}_{+}^{n}$ is a closed convex cone in $\mathbb{M}^{n}$. The interior of the positive semidefinite matrices is the positive definite matrices, for more basics on matrices see $[48,49]$. For $y, \mathfrak{z} \in \mathbb{R}^{n}$,

$$
\begin{aligned}
& y \leqq \mathfrak{z} \Longleftrightarrow y_{i} \leqq \mathfrak{z}_{i}, i=1, \ldots, n, \\
& y \leq \mathfrak{z} \Longleftrightarrow y \leqq \mathfrak{z}, y \neq \mathfrak{z}, \\
& y<\mathfrak{z} \Longleftrightarrow y_{i}<\mathfrak{z}_{i}, i=1, \ldots, n .
\end{aligned}
$$

Some index sets are as follows

$$
\begin{aligned}
M & =\left\{A \in \mathbb{M}_{+}^{n}: \mathscr{H}_{i}(A) \geqq 0, \mathscr{G}_{i}(A) \mathscr{H}_{i}(A) \leqq 0\right\}, \theta_{i}(A)=\mathscr{G}_{i}(A) \mathscr{H}_{i}(A), \\
\beth_{\mathfrak{f}} & =\{1, \ldots, p\}, \beth_{\mathfrak{f}}^{k}=\{1, \ldots, p\} \backslash\{k\}, \mathfrak{I}_{\mathscr{G}} \mathscr{H}:=\{1, \ldots, m\}, \\
Q & =\left\{A \in \mathbb{M}_{+}^{n}: \mathfrak{f}_{i}(A) \leqq \mathfrak{f}_{i}(\bar{A})\left(i \in \mathbb{I}_{\mathfrak{f}}\right), \mathscr{H}_{i}(A) \geqq 0, \mathscr{G}_{i}(A) \mathscr{H}_{i}(A) \leqq 0\right\}, \\
Q^{k} & =\left\{A \in \mathbb{M}_{+}^{n}: \mathfrak{f}_{i}(A) \leqq \mathfrak{f}_{i}(\bar{A})\left(i \in \beth_{\mathfrak{f}}^{k}\right), \mathscr{H}_{i}(A) \geqq 0, \mathscr{G}_{i}(A) \mathscr{H}_{i}(A) \leqq 0\right\}, \text { where } \bar{A} \in M, \\
\mathbb{R}_{+}^{n} & =\left\{x \in \mathbb{R}^{n}: x \geqq 0\right\}, \mathbb{R}_{++}^{n}=\left\{x \in \mathbb{R}^{n}: x>0\right\},
\end{aligned}
$$




$$
\begin{aligned}
& \beth_{0}=\beth_{0}(\bar{A}):=\left\{i \in I_{\mathscr{G} \mathscr{H}}: \mathscr{H}_{i}(\bar{A})=0\right\}, \beth_{+}=\beth_{+}(\bar{A}):=\left\{i \in I_{\mathscr{G}} \mathscr{H}: \mathscr{H}_{i}(\bar{A})>0\right\}, \\
& \beth_{0+}=\beth_{0+}(\bar{A}):=\left\{i \in \beth_{\mathscr{G} \mathscr{H}}: \mathscr{H}_{i}(\bar{A})=0, \mathscr{G}_{i}(\bar{A})>0\right\} \text {, } \\
& \beth_{00}=I_{00}(\bar{A}):=\left\{i \in I_{\mathscr{G} \mathscr{H}}: \mathscr{H}_{i}(\bar{A})=0, \mathscr{G}_{i}(\bar{A})=0\right\} \text {, } \\
& \beth_{0-}=\beth_{0-}(\bar{A}):=\left\{i \in I_{\mathscr{G}} \mathscr{H}: \mathscr{H}_{i}(\bar{A})=0, \mathscr{G}_{i}(\bar{A})<0\right\}, \\
& I_{+0}=I_{+0}(\bar{A}):=\left\{i \in I_{\mathscr{G} \mathscr{H}}: \mathscr{H}_{i}(\bar{A})>0, \mathscr{G}_{i}(\bar{A})=0\right\} \text {, } \\
& I_{+-}=I_{+-}(\bar{A}):=\left\{i \in I_{\mathscr{G} \mathscr{H}}: \mathscr{H}_{i}(\bar{A})>0, \mathscr{G}_{i}(\bar{A})<0\right\} .
\end{aligned}
$$

We discuss the solution concepts of $S-M M P V C$ motivated by Miettinen [34].

Definition 1. A feasible point $\bar{A}$ is said to be a weak efficient solution of $S-M M P V C$ if there is no any $A \in M$, such that

$$
\mathfrak{f}_{i}(A)<\mathfrak{f}_{i}(\bar{A}), \forall i \in \mathfrak{I}_{\mathfrak{f}} .
$$

Definition 2. A feasible point $\bar{A}$ is said to be a local weak efficient solution of $S-M M P V C$ if there exist a neighborhood $\mathscr{N}(\bar{A})$ of $\bar{A}$, such that there is no any $A \in M \cap \mathscr{N}(\bar{A})$, for which

$$
\mathfrak{f}_{i}(A)<\mathfrak{f}_{i}(\bar{A}), \forall i \in \beth_{\mathfrak{f}},
$$

holds.

Given a nonempty subset $M$ of $\mathbb{M}^{n}$, the closure, the convex hull and the convex cone (including the origin) generated by $M$ are denoted by $c l M, c o M$, and $c o n e M$, respectively. The negative and the strictly negative polar cone of $M$ are defined respectively by

$$
M^{-}:=\left\{V \in \mathbb{M}^{n}:\langle V, \mathscr{W}\rangle \leq 0, \forall \mathscr{W} \in M\right\}, M^{s}:=\left\{V \in \mathbb{M}^{n}:\langle V, \mathscr{W}\rangle<0, \forall \mathscr{W} \in M\right\}
$$

Contingent cone $T(M, A)$ to $M$ at point $A \in c l M$ are defined by

$$
T(M, A):=\left\{V \in \mathbb{M}^{n}: \exists t_{n} \downarrow 0, V_{n} \rightarrow V \text { such that } A+t_{n} V_{n} \in M \forall n\right\} .
$$

The notion of semi-regular convexificators [43] will be used here. It is observed that for locally Lipschitz function many generalized subdifferential like Clarke subdifferential [50], Michel-Penot subdifferential [51], Mordukhovich subdifferential [52], and Treiman subdifferential [53] are examples of upper semi-regular convexificators.

Let $\mathfrak{f}: \mathbb{M}^{n} \rightarrow \mathbb{R} \cup\{+\infty\}$ be an extended real-valued function and let $A \in \mathbb{M}^{n}$ at which $\mathfrak{f}$ is finite. The lower and upper Dini derivatives of $\mathfrak{f}$ at $A$ in the direction $V \in \mathbb{M}^{n}$ are defined, respectively, by

$$
\begin{aligned}
\mathfrak{f}^{-}(A ; V) & :=\liminf \inf _{t \downarrow 0} \frac{\mathfrak{f}(A+t V)-\mathfrak{f}(A)}{t}, \\
\mathfrak{f}^{+}(A ; V) & :=\lim \sup _{t \downarrow 0} \frac{\mathfrak{f}(A+t V)-\mathfrak{f}(A)}{t} .
\end{aligned}
$$

Now, we recall the definition of upper and lower semi-regular convexificators from [42,43].

Definition 3. Let $\mathfrak{f}: \mathbb{M}^{n} \rightarrow \mathbb{R} \cup\{+\infty\}$ be an extended real-valued function and let $A \in \mathbb{M}^{n}$ at which $\mathfrak{f}$ is finite. The function $\mathfrak{f}$ is said to admit an upper semi-regular convexificator $\partial^{*} \mathfrak{f}(A) \subset \mathbb{M}^{n}$ at $A$ if $\partial^{*} \mathfrak{f}(A)$ is closed and for each $V \in \mathbb{M}^{n}$,

$$
\mathfrak{f}^{+}(A ; V) \leq \sup _{\xi \in \partial^{*} \mathfrak{f}(A)}\langle\xi, V\rangle .
$$

The function $\mathfrak{f}$ is said to admit a lower semi-regular convexificator $\partial^{*} \mathfrak{f}(A) \subset \mathbb{M}^{n}$ at $A$ if $\partial^{*} \mathfrak{f}(A)$ is closed and for each $V \in \mathbb{M}^{n}$ 


$$
\mathfrak{f}^{-}(A ; V) \geq \inf _{\xi \in \partial^{*} \mathfrak{f}(A)}\langle\xi, V\rangle .
$$

Definition 4. Set $\partial \mathrm{f}(A)$ is said to be semi-regular convexificators if it satisfy both upper semiregular convexificators, as well as lower semi-regular convexificators.

Definition 5. Let $\mathfrak{f}: \mathbb{M}^{n} \rightarrow \mathbb{R} \cup\{+\infty\}$ be an extended real-valued function. Suppose that $A \in \mathbb{M}^{n}, \mathfrak{f}(A)$ is finite and admits a convexificator $\partial^{*} \mathfrak{f}(A)$ at $A$.

- $\quad \mathfrak{f}$ is said to be $\partial^{*}$-convex at $A$ if, and only if, for all $B \in \mathbb{M}^{n}$,

$$
\mathfrak{f}(B)-\mathfrak{f}(A) \geq\langle\xi, B-A\rangle, \forall \xi \in \partial^{*} \mathfrak{f}(A) .
$$

- $\quad \mathfrak{f}$ is said to be strictly $\partial^{*}$-convex at $A$ if, and only if, for all $B \in \mathbb{M}^{n}$,

$$
\mathfrak{f}(B)-\mathfrak{f}(A)>\langle\xi, B-A\rangle, \forall \xi \in \partial^{*} \mathfrak{f}(A) .
$$

- $\quad f$ is said to be $\partial^{*}$-pseudoconvex at $A$ if, and only if, for all $B \in \mathbb{M}^{n}$,

$$
\mathfrak{f}(B)<\mathfrak{f}(A) \Longrightarrow\langle\xi, B-A\rangle<0, \forall \xi \in \partial^{*} \mathfrak{f}(A) .
$$

- $\quad \mathfrak{f}$ is said to be strictly $\partial^{*}$-pseudoconvex at $A$ if, and only if, for all $B(\neq A) \in \mathbb{M}^{n}$,

$$
\langle\xi, B-A\rangle \geq 0 \Longrightarrow \mathfrak{f}(B)>\mathfrak{f}(A) \forall \xi \in \partial^{*} \mathfrak{f}(A) .
$$

- $\quad f$ is said to be $\partial^{*}-q u a s i c o n v e x$ at $A$ if, and only if, for all $B \in \mathbb{M}^{n}$,

$$
\mathfrak{f}(B) \leq \mathfrak{f}(A) \Longrightarrow\langle\xi, B-A\rangle \leq 0, \forall \xi \in \partial^{*} \mathfrak{f}(A) .
$$

Now, we recall generalized version of Farkas' lemma [54], which will play the vital role in the derivation of main result of this paper.

Lemma 1. (Farkas' Lemma) Let $\mathfrak{h}: \mathbb{M}^{n} \rightarrow \mathbb{R}^{m}$ be convex functions. Then, the following system:

$$
\left\{\begin{array}{l}
\mathfrak{h}(A)<0 \\
A \in M_{++}^{n}
\end{array}\right.
$$

has no solution if, and only if, there exists $(\lambda, \mathscr{W}) \in \mathbb{R}^{m} \times \mathbb{M}^{n}$ with $\lambda \geqq 0, \mathscr{W} \preceq 0$ and $(\lambda, \mathscr{W}) \neq$ $(0,0)$, such that

$$
\lambda^{T} \mathfrak{h}(A)+\langle\mathscr{W}, A\rangle \geqq 0, \forall A \in \mathbb{M}^{n} .
$$

\section{Optimality Conditions}

In this section, we deal with the traditional Fritz John necessary optimality conditions and propose some constraint qualifications to establish strong Karush-Kuhn-Tucker necessary optimality conditions, as well as sufficient optimality conditions for semidefinite multiobjective mathematical programs with vanishing constraints in terms of convexificators.

Theorem 1. (Fritz-John necessary optimality conditions) Let $\bar{A}$ be a local weak efficient solution for $(S-M M P V C)$. Suppose that $\mathfrak{f}_{i}\left(i \in I_{\mathfrak{f}}\right)$ and $\mathscr{H}_{i}\left(i \in \beth_{0}\right), \mathscr{G}_{i}\left(i \in I_{+0}\right)$, admit bounded upper semi-regular convexificators and for each $\mathscr{H}_{i}\left(i \in I_{+}\right), \mathscr{G}_{i}\left(i \in \beth_{0} \cup I_{+-}\right)$, is continuous. Then, there exist $\bar{\lambda}_{i}^{\mathfrak{f}} \geqq 0\left(i \in \beth_{\mathfrak{f}}\right), \bar{\lambda}_{i}^{\mathscr{H}} \geqq 0\left(i \in \beth_{0-} \cup \beth_{00}\right), \bar{\lambda}_{i}^{\mathscr{H}}$ free $\left(i \in \beth_{0+}\right), \bar{\lambda}_{i}^{\mathscr{G}} \geqq$ $0\left(i \in I_{+0}\right), \bar{\lambda}_{i}^{\mathscr{G}}=0\left(i \in \mathbf{I}_{0} \cup \beth_{+-}\right), \overline{\mathscr{W}} \in \mathbb{M}_{+}^{n}$ and not all multipliers along with $\overline{\mathscr{W}}$ can be simultaneously zero, such that

$$
0 \in \sum_{i=1}^{p} \bar{\lambda}_{i}^{\mathfrak{f}} \operatorname{cod}^{*} \mathfrak{f}_{i}(\bar{A})+\sum_{i=1}^{m}\left[\bar{\lambda}_{i}^{\mathscr{G}} \cot ^{*} \mathscr{G}_{i}(\bar{A})-\bar{\lambda}_{i}^{\mathscr{H}} \operatorname{cod}^{*} \mathscr{H}_{i}(\bar{A})\right]-\overline{\mathscr{W}},\langle\bar{A}, \overline{\mathscr{W}}\rangle=0 .
$$


Proof. We have to show that

$$
\begin{aligned}
\left(\left(\bigcup_{i \in I_{\mathfrak{f}}} \partial^{*} \mathfrak{f}_{i}(\bar{A})\right)^{s}+\bar{A}\right) & \bigcap\left(\left(\bigcup_{i \in I_{0+} \cup \beth_{00} \cup \beth_{0-}}-\partial^{*} \mathscr{H}_{i}(\bar{A})\right)^{s}+\bar{A}\right) \\
& \bigcap\left(\left(\bigcup_{i \in I_{0+} \cup \beth_{00} \cup \beth_{0_{-}} \cup I_{+0}} \partial^{*} \theta_{i}(\bar{A})\right)^{s}+\bar{A}\right) \bigcap M_{++}^{n}=\varnothing .
\end{aligned}
$$

Suppose, on the contrary,

$$
\begin{aligned}
A \in\left(\left(\bigcup_{i \in \beth_{\mathfrak{f}}} \partial^{*} f_{i}(\bar{A})\right)^{s}+\bar{A}\right) & \bigcap\left(\left(\bigcup_{i \in I_{0+} \cup \beth_{00} \cup \beth_{0-}}-\partial^{*} \mathscr{H}_{i}(\bar{A})\right)^{s}+\bar{A}\right) \\
& \bigcap\left((\underbrace{}_{i \in I_{0+} \cup \beth_{00} \cup \beth_{0-} \cup I_{+0}} \partial^{*} \theta_{i}(\bar{A}))^{s}+\bar{A}\right) \bigcap M_{++}^{n} .
\end{aligned}
$$

As, $\mathfrak{f}_{i}\left(i \in I_{\mathfrak{f}}\right), \mathscr{H}_{i}\left(i \in \beth_{0+} \cup \beth_{00} \cup \beth_{0_{-}}\right)$and $\theta_{i}\left(i \in \beth_{+0} \cup \beth_{00} \cup \beth_{0-} \cup \beth_{0+}\right)$, admit bounded upper semi-regular convexificators, we deduce that

$$
\begin{aligned}
\mathfrak{f}_{i}^{+}(\bar{A}, A-\bar{A}) & <0, i \in \beth_{\mathfrak{f},} \\
-\mathscr{H}_{i}^{+}(\bar{A}, A-\bar{A}) & <0, i \in \beth_{0+} \cup \beth_{00} \cup \beth_{0-}, \\
\theta_{i}^{+}(\bar{A}, A-\bar{A}) & <0, i \in \beth_{0+} \cup \beth_{00} \cup \beth_{0-} \cup \beth_{+0} .
\end{aligned}
$$

Therefore, there exists $\tau>0$ and $t \in(0, \tau)$ such that

$$
\begin{aligned}
\mathfrak{f}_{i}(\bar{A}+t(A-\bar{A})) & <\mathfrak{f}_{i}(\bar{A}), i \in \beth_{\mathfrak{f}}, \\
-\mathscr{H}_{i}(\bar{A}+t(A-\bar{A})) & <-\mathscr{H}_{i}(\bar{A}), i \in \beth_{0+} \cup \beth_{00} \cup \beth_{0-}, \\
\theta_{i}(\bar{A}+t(A-\bar{A})) & <\theta_{i}(\bar{A}), i \in \beth_{0+} \cup \beth_{00} \cup \beth_{0-} \cup \beth_{+0} .
\end{aligned}
$$

The continuity of $\mathscr{H}_{i}\left(i \in I_{+-} \cup \beth_{+0}\right)$ and $\theta_{i}\left(i \in I_{+-}\right)$implies there exists $\tau>0$, such that $\forall t \in(0, \tau)$,

$$
-\mathscr{H}_{i}(\bar{A}+t(A-\bar{A}))<0\left(i \in \beth_{+-} \cup \beth_{+0}\right), \theta_{i}(\bar{A}+t(A-\bar{A}))<0\left(i \in I_{+-}\right) .
$$

From (4)-(7) and the convexity of $M_{+}^{n}$ we find the contradiction with the local weak efficient point of $\bar{A}$. Consider

$$
\begin{aligned}
& \phi_{i}(A)=\sup _{\xi_{i} \in \partial^{*} \mathfrak{f}_{i}(\bar{A})}\left\langle\xi_{i}, A-\bar{A}\right\rangle, i \in \beth_{\mathfrak{f}}, \\
& \psi_{i}(A)=\sup _{\eta_{i} \in-\partial^{*} \mathscr{H}_{i}(\bar{A})}\left\langle\eta_{i}, A-\bar{A}\right\rangle, i \in \beth_{0+} \cup \beth_{00} \cup \beth_{0-}, \\
& \varphi_{i}(A)=\sup _{\zeta_{i} \in \partial^{*} \theta_{i}(\bar{A})}\left\langle\zeta_{i}, A-\bar{A}\right\rangle, i \in \beth_{0+} \cup \beth_{00} \cup \beth_{0-} \cup \beth_{+0} .
\end{aligned}
$$

Easily, we can seen that $\phi_{i}(\cdot), \psi_{i}(\cdot)$ and $\varphi_{i}(\cdot)$ are convex functions. From (2), it follows that the following system has no solution

$$
K= \begin{cases}\phi_{i}(A)<0 \quad \text { if } i \in \beth_{\mathfrak{f}} \\ \psi_{i}(A)<0 \text { if } i \in \beth_{0+} \cup \beth_{00} \cup \beth_{0-}, \\ \varphi_{i}(A)<0 \text { if } i \in \beth_{0+} \cup \beth_{00} \cup \beth_{0-} \cup \beth_{+0}, \\ M_{++}^{n} \cdot\end{cases}
$$


Farkas' Lemma 1 implies that there exist $\bar{\lambda}_{i}^{\mathfrak{f}} \geqq 0\left(i \in \mathbf{I}_{\mathfrak{f}}\right), \lambda_{i}^{\mathscr{H}} \geqq 0\left(i \in \beth_{0+} \cup \beth_{00} \cup\right.$ $\left.I_{0-}\right), \lambda_{i}^{\theta} \geqq 0\left(i \in I_{0+} \cup I_{00} \cup I_{0-} \cup I_{+0}\right)$ and $\overline{\mathscr{W}} \in M_{+}^{n}$ and not all multipliers along with $\overline{\mathscr{W}}$ can be simultaneously zero, such that

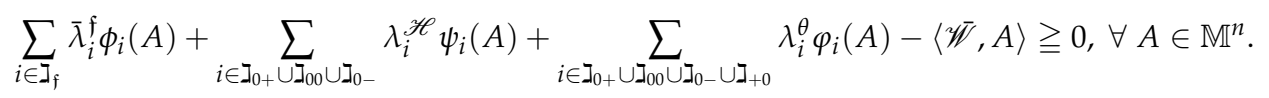

The above inequality (8) implies that $\langle\overline{\mathscr{W}}, \bar{A}\rangle \leqq 0$. Differently, $\overline{\mathscr{W}}$ and $\bar{A}$ are two elements in $\mathbb{M}_{+}^{n}$, hence $\langle\overline{\mathscr{V}}, \bar{A}\rangle=0$. Therefore,

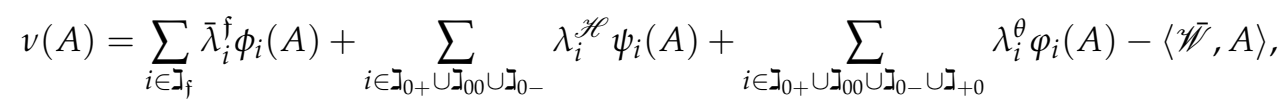

is a convex function and $v(\bar{A})=0$. This implies $0 \in \partial v(\bar{A})$, where $\partial v(\bar{A})$ is the subdifferential set for $v$. Hence,

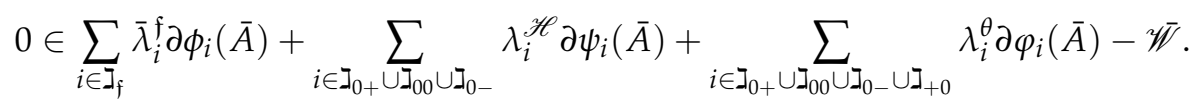

This implies,

$$
\begin{aligned}
& 0 \in \sum_{i \in \beth_{\mathfrak{f}}} \bar{\lambda}_{i}^{\mathfrak{f}} \partial^{*} \phi_{i}(\bar{A})+\sum_{i \in \beth_{0+} \cup \beth_{00} \cup \beth_{0_{-}}} \lambda_{i}^{\mathscr{H}} \partial^{*} \psi_{i}(\bar{A})+\sum_{i \in I_{0+} \cup \sum_{00} \cup \beth_{I_{0}} \cup I_{+0}} \lambda_{i}^{\theta} \partial^{*} \varphi_{i}(\bar{A})-\overline{\mathscr{W}} .
\end{aligned}
$$

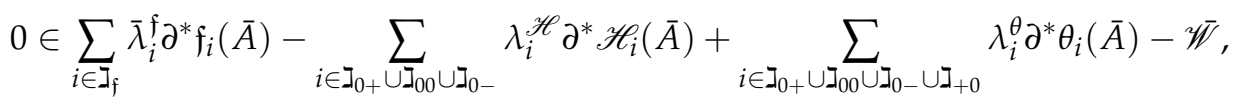

$$
\begin{aligned}
& 0 \in \sum_{i \in I_{\mathfrak{f}}} \bar{\lambda}_{i}^{\mathfrak{f}} \partial^{*} \mathfrak{f}_{i}(\bar{A})-\sum_{i \in \beth_{0+} \cup \beth_{00} \cup \beth_{0-}} \lambda_{i}^{\mathscr{H}} \partial^{*} \mathscr{H}{ }_{i}(\bar{A}) \\
& +\sum_{i \in \beth_{0+} \cup \beth_{00} \cup \beth_{\mathbb{I}_{-}} \cup \beth_{+0}} \lambda_{i}^{\theta}\left[\mathscr{H}_{i}(\bar{A}) \partial^{*} \mathscr{G}_{i}(\bar{A})+\mathscr{G}_{i}(\bar{A}) \partial^{*} \mathscr{H}_{i}(\bar{A})\right]-\mathscr{\mathscr { W }} .
\end{aligned}
$$

For $\lambda_{i}^{\mathscr{H}}=0\left(i \in \mathbf{I}_{+-} \cup \boldsymbol{I}_{+0}\right), \lambda_{i}^{\theta}=0\left(i \in \mathbf{I}_{+-}\right)$, we obtain from (9)

$$
\begin{aligned}
& 0 \in \sum_{i \in \beth_{f}} \bar{\lambda}_{i}^{f} \operatorname{cod} \partial^{*} f_{i}(\bar{A})+\sum_{i=1}^{m}\left[\bar{\lambda}_{i}^{\mathscr{G}} \cot ^{*} \mathscr{G}_{i}(\bar{A})-\bar{\lambda}_{i}^{\mathscr{H}} \operatorname{co} \partial^{*} \mathscr{H}_{i}(\bar{A})\right]-\overline{\mathscr{W}}, \\
& \text { where } \bar{\lambda}_{i}^{\mathscr{H}}=\lambda_{i}^{\mathscr{H}}-\lambda_{i}^{\theta} \mathscr{G}_{i}(\bar{A})\left(i \in \beth_{0+} \cup \beth_{0-} \cup \beth_{00} \cup \beth_{+0}\right), \\
& \bar{\lambda}_{i}^{\mathscr{H}}=\lambda_{i}^{\theta}=0\left(i \in \beth_{+-}\right), \bar{\lambda}_{i}^{\mathscr{G}}=\lambda^{\theta} \mathscr{H}_{i}(\bar{A})\left(i \in \beth_{0+} \cup \beth_{0-} \cup \beth_{00} \cup \beth_{+0}\right), \\
& \bar{\lambda}_{i}^{\mathscr{G}}=\lambda_{i}^{\theta}=0\left(i \in \beth_{+-}\right) .
\end{aligned}
$$

Thus, we have

$$
\begin{aligned}
& 0 \in \sum_{i \in \mathcal{I}_{\mathfrak{f}}} \bar{\lambda}_{i}^{\mathfrak{f}} \operatorname{co\partial ^{*}} \mathfrak{f}_{i}(\bar{A})+\sum_{i=1}^{m}\left[\bar{\lambda}_{i}^{\mathscr{G}} \cot ^{*} \mathscr{G}_{i}(\bar{A})-\bar{\lambda}_{i}^{\mathscr{H}} \operatorname{cod}^{*} \mathscr{H}_{i}(\bar{A})\right]-\overline{\mathscr{W}}, \\
& \bar{\lambda}_{i}^{\mathfrak{f}} \geqq 0\left(i \in \mathrm{I}_{\mathfrak{f}}\right),\langle\overline{\mathscr{W}}, \bar{A}\rangle=0, \bar{\lambda}_{i}^{\mathscr{H}}=0\left(i \in \mathrm{I}_{+0} \cup \mathrm{I}_{+-}\right), \bar{\lambda}_{i}^{\mathscr{H}} \geqq 0\left(i \in \mathrm{I}_{0_{-}} \cup \mathrm{I}_{00}\right), \bar{\lambda}_{i}^{\mathscr{H}} \text { free }\left(i \in \mathrm{I}_{0+}\right), \\
& \bar{\lambda}_{i}^{\mathscr{G}}=0\left(i \in \mathrm{I}_{0+} \cup \mathrm{I}_{0-} \cup \mathrm{I}_{00} \cup \mathrm{I}_{+-}\right), \bar{\lambda}_{i}^{\varphi} \geqq 0\left(i \in \mathrm{I}_{+0}\right) .
\end{aligned}
$$

Definition 6. The generalized Cottle constraint qualification (GCCQ) is said to satisfy at $\bar{A}$ if

$$
\left(\bigcup_{i \in \beth_{\mathfrak{f}}^{k}} \cot ^{*} \mathfrak{f}_{i}(\bar{A})\right)^{s} \cap\left(\bigcup_{i \in I_{0+}} \operatorname{cod}^{*} \mathscr{H}_{i}(\bar{A}) \bigcup_{i \in I_{0+}}-\operatorname{co}^{*} \mathscr{H}_{i}(\bar{A})\right.
$$




$$
\left.\bigcup_{i \in \beth_{0}-\cup \beth_{00}}-\cot ^{*} \mathscr{H}_{i}(\bar{A}) \bigcup_{i \in I_{+0}} \cot ^{*} \mathscr{G}_{i}(\bar{A})\right)^{s} \bigcap M_{+}^{n} \neq \varnothing, \forall k \in \beth_{\mathfrak{f}} .
$$

Theorem 2. Let $\bar{A}$ be a local weak efficient solution for $(S-M M P V C)$. Suppose that $\mathfrak{f}_{i}$ $\left(i \in I_{\mathfrak{f}}\right), \mathscr{H}_{i}\left(i \in I_{0}\right)$ and $\mathscr{G}_{i}\left(i \in I_{+0}\right)$ admit bounded upper semi-regular convexificators and $\mathscr{H}_{i}\left(i \in I_{+}\right), \mathscr{G}_{i}\left(i \in I_{0} \cup I_{+-}\right)$are continuous. If (GCCQ) holds at $\bar{A}$ then there exist $\bar{\lambda}_{i}^{\mathfrak{f}}>0\left(i \in \mathbf{I}_{\mathfrak{f}}\right), \bar{\lambda}_{i}^{\mathscr{H}}, \bar{\lambda}_{i}^{\mathscr{G}} \in \mathbb{R}^{m}, \mathscr{\mathscr { W }} \in \mathbb{M}_{+}^{n}$, such that

$$
\begin{array}{r}
0 \in \sum_{i \in \beth_{\mathfrak{f}}} \bar{\lambda}_{i}^{\mathfrak{f}} \operatorname{cod}^{*} \mathfrak{f}_{i}(\bar{A})+\sum_{i=1}^{m}\left[\bar{\lambda}_{i}^{\mathscr{G}} \operatorname{cod}^{*} \mathscr{G}_{i}(\bar{A})-\bar{\lambda}_{i}^{\mathscr{H}} \operatorname{cod}^{*} \mathscr{H}_{i}(\bar{A})\right]-\overline{\mathscr{W}}, \\
\langle\overline{\mathscr{W}}, \bar{A}\rangle=0, \bar{\lambda}_{i}^{\mathscr{H}}=0\left(i \in \beth_{+0} \cup \beth_{+-}\right), \bar{\lambda}_{i}^{\mathscr{H}} \geqq 0\left(i \in \beth_{0-} \cup \beth_{00}\right), \bar{\lambda}_{i}^{\mathscr{H}} \text { free }\left(i \in \beth_{0+}\right), \\
\bar{\lambda}_{i}^{\mathscr{G}}=0\left(i \in \beth_{0+} \cup \beth_{0-} \cup \beth_{00} \cup \beth_{+-}\right), \bar{\lambda}_{i}^{\mathscr{G}} \geqq 0\left(i \in \beth_{+0}\right) .
\end{array}
$$

Proof. Since $\bar{A}$ is a local weak efficient solution, Theorem 1 implies that there exist $\bar{\lambda}_{i}^{\mathfrak{f}} \geqq 0\left(i \in \beth_{\mathfrak{f}}\right), \bar{\lambda}_{i}^{\mathscr{H}} \geqq 0, \bar{\lambda}_{i}^{\mathscr{G}} \geqq 0$ and $\overline{\mathscr{W}} \in \mathbb{M}_{+}^{n}$, such that

$$
\begin{aligned}
& 0 \in \sum_{i \in \beth_{\mathfrak{f}}} \bar{\lambda}_{i}^{\mathfrak{f}} \operatorname{cod}^{*} \mathfrak{f}_{i}(\bar{A})+\sum_{i=1}^{m}\left[\bar{\lambda}_{i}^{\mathscr{G}} \cot ^{*} \mathscr{G}_{i}(\bar{A})-\bar{\lambda}_{i}^{\mathscr{H}} \operatorname{cod}^{*} \mathscr{H}_{i}(\bar{A})\right]-\overline{\mathscr{W}}, \\
& \langle\overline{\mathscr{W}}, \bar{A}\rangle=0, \bar{\lambda}_{i}^{\mathscr{H}}=0\left(i \in \beth_{+0} \cup \beth_{+-}\right), \bar{\lambda}_{i}^{\mathscr{H}} \geqq 0\left(i \in \beth_{0-} \cup \beth_{00}\right), \bar{\lambda}_{i}^{\mathscr{H}} \text { free }\left(i \in \beth_{0+}\right) \text {, } \\
& \bar{\lambda}_{i}^{\mathscr{G}}=0\left(i \in \beth_{0+} \cup \beth_{0_{-}} \cup \beth_{00} \cup I_{+-}\right), \bar{\lambda}_{i}^{\mathscr{G}} \geqq 0\left(i \in I_{+0}\right) .
\end{aligned}
$$

Without loss of generality, assume that $\lambda_{1}=0$, then there exist $\xi_{i} \in \operatorname{co\partial f_{i}}(\bar{A})\left(i \in \beth_{\mathfrak{f}}^{1}\right)$, $\eta_{i} \in \operatorname{co\partial } \mathscr{H}_{i}(\bar{A}), \zeta_{i} \in \operatorname{co\partial } \mathscr{G}_{i}(\bar{A})$, such that Equation (11) becomes

$$
0=\sum_{i \in \Xi_{\mathfrak{f}}^{1}} \bar{\lambda}_{i}^{\mathfrak{f}} \xi_{i}+\sum_{i=1}^{m}\left[\bar{\lambda}_{i}^{\mathscr{G}} \zeta_{i}-\bar{\lambda}_{i}^{\mathscr{H}} \eta_{i}\right]-\overline{\mathscr{W}}
$$

it follows from (GCCQ), there exists $A \in \mathbb{M}_{+}^{n}$ such that

$$
\begin{array}{r}
0>\sum_{i \in]_{\mathfrak{f}}^{1}} \bar{\lambda}_{i}^{\mathfrak{f}}\left\langle\xi_{i}, A\right\rangle+\sum_{i=1}^{m}\left[\bar{\lambda}_{i}^{\mathscr{G}}\left\langle\zeta_{i}, A\right\rangle-\bar{\lambda}_{i}^{\mathscr{H}}\left\langle\eta_{i}, A\right\rangle\right]-\langle\overline{\mathscr{W}}, A\rangle \\
=\left\langle\sum_{i \in \mathrm{J}_{\mathfrak{f}}^{1}} \bar{\lambda}_{i}^{\mathfrak{f}} \xi_{i}+\sum_{i=1}^{m}\left[\bar{\lambda}_{i}^{\mathscr{G}} \zeta_{i}-\bar{\lambda}_{i}^{\mathscr{H}} \eta_{i}\right]-\mathscr{\mathscr { W }}, A\right\rangle=0 .
\end{array}
$$

This contradicts the assumption. Thus, we obtain $\lambda_{1}^{f}>0$. Repeating the above process for each $k \in I_{\mathfrak{f}}$ we find the required result.

Now, we introduce more relaxed constraint qualifications than (GCCQ).

Definition 7. The generalized Guignard constraint qualification (GGCQ) is said to be hold at $\bar{A}$ if

$$
\begin{aligned}
& C=\operatorname{coneco}\left(\bigcup_{i \in I_{0+}} \cot ^{*} \mathscr{H}_{i}(\bar{A}) \bigcup_{i \in I_{0+}}-\cot ^{*} \mathscr{H}_{i}(\bar{A})\right. \\
& \left.\bigcup_{i \in I_{0-} \cup \beth_{00}}-\cot ^{*} \mathscr{H}_{i}(\bar{A}) \bigcup_{i \in I_{+0}} \cot ^{*} \mathscr{G}_{i}(\bar{A})\right)-\mathbb{M}_{+}^{n} \text { is closed set and } \\
& \left(\bigcup_{i \in I_{f}} \cot ^{*} \mathfrak{f}_{i}(\bar{A})\right)^{-} \cap\left(\bigcup_{i \in I_{0+}} \cot ^{*} \mathscr{H}_{i}(\bar{A}) \bigcup_{i \in \beth_{0+}}-\cot ^{*} \mathscr{H}_{i}(\bar{A})\right.
\end{aligned}
$$




$$
\left.\bigcup_{i \in I_{0}-\cup I_{00}}-\cot ^{*} \mathscr{H}(\bar{A}) \bigcup_{i \in I_{+0}} \operatorname{coz}^{*} \mathscr{G}_{i}(\bar{A})\right)^{-} \bigcap M_{+}^{n} \subset \bigcap_{i=1}^{p} \operatorname{coT}\left(Q^{i}, \bar{A}\right) .
$$

Lemma 2. Let $\bar{A}$ be any feasible solution to problem $(S-M M P V C)$. Suppose that $\mathfrak{f}_{i}\left(i \in \mathbb{I}_{\mathfrak{f}}\right)$, $\mathscr{H}_{i}\left(i \in \beth_{0}\right), \mathscr{G}_{i}\left(i \in \beth_{+0}\right)$, admit bounded upper semi-regular convexificators and for each $\mathscr{H}_{i}\left(i \in I_{+}\right), \mathscr{G}_{i}\left(i \in \beth_{0} \cup I_{+-}\right)$, are continuous. If $C$ is closed and GCCQ holds at $\bar{A}$, then GGCQ holds at $\bar{A}$.

Proof. Without loss of generality, we assume that $A$ satisfies GCCQ for $k=1$.

$$
\begin{array}{r}
A \in\left(\bigcup_{i \in \beth_{f}^{1}} \operatorname{cod}^{*} \mathfrak{f}_{i}(\bar{A})\right)^{-} \cap\left(\bigcup_{i \in \beth_{0+}} \cot ^{*} \mathscr{H}_{i}(\bar{A}) \bigcup_{i \in \beth_{0+}}-\operatorname{cod}^{*} \mathscr{H}_{i}(\bar{A})\right. \\
\left.\bigcup_{i \in \beth_{0-} \cup \beth_{00}}-\cot ^{*} \mathscr{H}_{i}(\bar{A}) \bigcup_{i \in I_{+0}} \cot ^{*} \mathscr{G}_{i}(\bar{A})\right)^{-} \bigcap M_{+}^{n} \neq \varnothing .
\end{array}
$$

Since all $\mathfrak{f}_{i}\left(i \in \beth_{\mathfrak{f}}\right), \mathscr{H}_{i}\left(i \in \beth_{0}\right), \mathscr{G}_{i}\left(i \in \beth_{+0}\right)$, admit bounded upper semi-regular convexificators, so we have

$$
\begin{array}{r}
\mathfrak{f}_{i}^{+}(\bar{A} ; A)<0, \forall i \in \mathbb{I}_{\mathfrak{f}}^{1}, \\
-\mathscr{H}_{i}^{+}(\bar{A} ; A)<0, \forall i \in I_{0}, \\
\mathscr{G}_{i}^{+}(\bar{A} ; A)<0, \forall i \in I_{+0} .
\end{array}
$$

Since $\mathbb{M}_{+}^{n}$ is a convex cone, there exists $\tau>0$, such that

$$
\begin{aligned}
& \mathfrak{f}_{i}(\bar{A}+t A)<\mathfrak{f}_{i}(\bar{A})\left(i \in \mathbf{I}_{\mathfrak{f}}^{1}\right),-\mathscr{H}_{i}(\bar{A}+t A)<0, \forall i \in I_{0}, \mathscr{G}_{i}(\bar{A}+t A)<0, \forall i \in \beth_{+0}, \\
& \bar{A}+t A \in \mathbb{M}_{+}^{n} \forall t \in(0, \tau) .
\end{aligned}
$$

On the other hand $\mathscr{H}_{i}\left(i \in I_{+}\right), \mathscr{G}_{i}\left(i \in I_{0} \cup I_{+-}\right)$are a continuous. Therefore, there exists $\tau>0$, such that

$$
-\mathscr{H}_{i}(\bar{A}+t A)<0\left(i \in \mathbf{I}_{+}\right), \mathscr{G}_{i}(\bar{A}+t A)<0\left(i \in I_{0} \cup I_{+-}\right) \bar{A}+t A \in \mathbb{M}_{+}^{n}, t \in(0, \tau) .
$$

Thus, $A \in T\left(Q^{1}, \bar{A}\right)$. Therefore, we have

$$
\begin{aligned}
& \mathscr{A}=\left(\bigcup_{i \in \beth_{f}} \operatorname{cod}^{*} f_{i}(\bar{A})\right)^{-} \bigcap\left(\bigcup_{i \in \beth_{0+}} \cot ^{*} \mathscr{H}_{i}(\bar{A}) \bigcup_{i \in \beth_{0+}}-\operatorname{cod}^{*} \mathscr{H}_{i}(\bar{A})\right. \\
& \left.\bigcup_{i \in I_{0-} \cup \beth_{00}}-\operatorname{cod}^{*} \mathscr{H}_{i}(\bar{A}) \bigcup_{i \in I_{+0}} \operatorname{cod}^{*} \mathscr{G}_{i}(\bar{A})\right)^{-} \bigcap M_{+}^{n} \\
& =\operatorname{cl}\left(( \bigcup _ { i \in \Xi _ { f } } \operatorname { c o d } ^ { * } \mathfrak { f } _ { i } ( \overline { A } ) ) ^ { s } \cap \left(\bigcup_{i \in \beth_{0+}} \cot ^{*} \mathscr{H}_{i}(\bar{A}) \bigcup_{i \in \beth_{0+}}-\operatorname{cod}^{*} \mathscr{H}_{i}(\bar{A})\right.\right. \\
& \left.\left.\bigcup_{i \in \beth_{0} \cup \cup \beth_{00}}-\operatorname{cod}^{*} \mathscr{H}_{i}(\bar{A}) \bigcup_{i \in \beth_{+0}} \operatorname{cod}^{*} \mathscr{G}_{i}(\bar{A})\right)^{s} \bigcap M_{++}^{n}\right) \\
& \subset \operatorname{cl}\left(( \bigcup _ { i \in \beth _ { f } ^ { 1 } } \operatorname { c o d } ^ { * } \mathfrak { f } _ { i } ( \overline { A } ) ) ^ { s } \cap \left(\bigcup_{i \in I_{0+}} \operatorname{cod}^{*} \mathscr{H}_{i}(\bar{A}) \bigcup_{i \in I_{0+}}-\operatorname{cod}^{*} \mathscr{H}_{i}(\bar{A})\right.\right. \\
& \left.\left.\bigcup_{i \in I_{0-} \cup I_{00}}-\cot ^{*} \mathscr{H}_{i}(\bar{A}) \bigcup_{i \in I_{+0}} \cot ^{*} \mathscr{G}_{i}(\bar{A})\right)^{s} \bigcap M_{++}^{n}\right)
\end{aligned}
$$




$$
\subset \operatorname{clcoT}\left(Q^{1}, \bar{A}\right)=\operatorname{coT}\left(Q^{1}, \bar{A}\right) .
$$

Similarly, it can be proved that $\mathscr{A} \subset \operatorname{coT}\left(Q^{i}, \bar{A}\right), \forall i \in \beth_{\mathfrak{f}}$. Therefore

$$
\begin{aligned}
& \left(\bigcup_{i \in \Xi_{\mathcal{f}}} \operatorname{cod}^{*} \mathfrak{f}_{i}(\bar{A})\right)^{-} \cap\left(\bigcup_{i \in I_{0+}} \cot ^{*} \mathscr{H}_{i}(\bar{A}) \bigcup_{i \in \beth_{0+}}-\cot \partial^{*} \mathscr{H}_{i}(\bar{A})\right. \\
& \left.\bigcup_{i \in \beth_{0} \cup \beth_{00}}-\operatorname{cod}^{*} \mathscr{H}_{i}(\bar{A}) \bigcup_{i \in I_{+0}} \operatorname{cod}^{*} \mathscr{G}_{i}(\bar{A})\right)^{-} \bigcap M_{+}^{n} \subset \bigcap_{i=1}^{p} \operatorname{coT} T\left(Q^{i}, \bar{A}\right) .
\end{aligned}
$$

We present an example to show that converse of the above Lemma (2) does not hold.

Example 1. Consider the problem

$$
\begin{array}{r}
\min \left(\mathfrak{f}_{1}(A), \mathfrak{f}_{2}(A)\right) \text {, subject to } \mathscr{H}(A)=x_{1} \geqq 0, \mathscr{G}(A) \mathscr{H}(A)=x_{3} . x_{1} \leqq 0, \\
A=\left[\begin{array}{ll}
x_{1} & x_{2} \\
x_{2} & x_{3}
\end{array}\right] \in \mathbb{M}_{+}^{2} \text {, where } \mathfrak{f}_{1}(A)=\left|x_{1}\right|, \mathfrak{f}_{2}(A)=\left|x_{3}\right| .
\end{array}
$$

Feasible set $M=\left\{\left[\begin{array}{ll}x_{1} & x_{2} \\ x_{2} & x_{3}\end{array}\right] \in \mathbb{M}_{+}^{2}: x_{1} \geqq 0, x_{1} x_{3} \leqq 0\right\}$. Since $\bar{A}=\left[\begin{array}{ll}0 & 0 \\ 0 & 0\end{array}\right]$, is weak efficient solution for the considered problem. Now, we can find upper semi-regular convexificator of each functions at point $\bar{A}$ as follows:

$$
\begin{gathered}
\partial^{*} \mathfrak{f}_{1}(\bar{A})=\left\{\left[\begin{array}{cc}
-1 & 0 \\
0 & 0
\end{array}\right],\left[\begin{array}{ll}
1 & 0 \\
0 & 0
\end{array}\right]\right\}, \partial^{*} \mathfrak{f}_{2}(\bar{A})=\left\{\left[\begin{array}{cc}
0 & 0 \\
0 & -1
\end{array}\right],\left[\begin{array}{ll}
0 & 0 \\
0 & 1
\end{array}\right]\right\}, \\
\partial^{*} \mathscr{H}(\bar{A})=\left\{\left[\begin{array}{ll}
1 & 0 \\
0 & 0
\end{array}\right]\right\}, \partial^{*} \mathscr{G}(\bar{A})=\left\{\left[\begin{array}{ll}
0 & 0 \\
0 & 1
\end{array}\right]\right\} . \\
Q^{1}=\left\{\left[\begin{array}{ll}
x_{1} & x_{2} \\
x_{2} & x_{3}
\end{array}\right] \in \mathbb{M}_{+}^{2}: x_{1} \geqq 0, x_{2}=0, x_{3}=0\right\}, \\
Q^{2}=\left\{\left[\begin{array}{ll}
x_{1} & x_{2} \\
x_{2} & x_{3}
\end{array}\right] \in \mathbb{M}_{+}^{2}: x_{1}=0, x_{2}=0, x_{3} \in \mathbb{R}\right\} .
\end{gathered}
$$

So, we conclude that

$$
\left[\begin{array}{ll}
0 & 0 \\
0 & 0
\end{array}\right] \in \bigcap_{i=1}^{2} \operatorname{coT}\left(Q^{i}, \bar{A}\right) \text { and } \bigcup_{i=1}^{2} \cot ^{*} \mathfrak{f}_{i}(\bar{A})=\left\{\left[\begin{array}{ll}
t & 0 \\
0 & 0
\end{array}\right],\left[\begin{array}{ll}
0 & 0 \\
0 & s
\end{array}\right]: t, s \in[-1,1]\right\},
$$

thus, we have

$$
\left(\bigcup_{i=1}^{2} \cot ^{*} \mathfrak{f}_{i}(\bar{A})\right)^{-}=\left\{\left[\begin{array}{cc}
0 & x_{2} \\
x_{2} & 0
\end{array}\right]: x_{2} \in \mathbb{R}\right\}
$$

Since,

$$
\cot ^{*} \mathscr{H}(\bar{A})=\left\{\left[\begin{array}{ll}
1 & 0 \\
0 & 0
\end{array}\right]\right\} \text {, then }\left(-\cot ^{*} \mathscr{H}(\bar{A})\right)^{-}=\left\{\left[\begin{array}{ll}
x_{1} & x_{2} \\
x_{2} & x_{3}
\end{array}\right]: x_{1} \geqq 0\right\} .
$$


Consequently, we have

$$
\left(\bigcup_{i=1}^{2} \operatorname{cod}^{*} \mathfrak{f}_{i}(\bar{A})\right)^{-} \bigcap\left(-\operatorname{cod}^{*} \mathscr{H}(\bar{A})\right)^{-} \bigcap \mathbb{M}_{+}^{2}=\left\{\left[\begin{array}{ll}
0 & 0 \\
0 & 0
\end{array}\right]\right\} \subset \bigcap_{i=1}^{2} \cot \left(Q^{i}, \bar{A}\right) .
$$

Obviously, $C=$ cone $\cot ^{*} \mathscr{H}(\bar{A})-\mathbb{M}_{+}^{2}$ is closed set. Hence, (GGCQ) satisfied at $\bar{A}$. Now,

$$
\left(\bigcup_{i \in \mathbb{J}_{\mathfrak{f}}^{1}} \operatorname{cod}^{*} \mathfrak{f}_{i}(\bar{A})\right)^{s}=\left(\operatorname{cod}^{*} \mathfrak{f}_{2}(\bar{A})\right)^{s}=\varnothing,\left(\bigcup_{i \in \mathbb{J}_{\mathfrak{f}}^{2}} \operatorname{cod}^{*} \mathfrak{f}_{i}(\bar{A})\right)^{s}=\left(\operatorname{cod}^{*} \mathfrak{f}_{1}(\bar{A})\right)^{s}=\varnothing,
$$

which implies that

$$
\begin{aligned}
& \left(\bigcup_{i \in \beth_{\mathfrak{f}}^{k}} \operatorname{co\partial }^{*} \mathfrak{f}_{i}(\bar{A})\right)^{s} \cap\left(\bigcup_{i \in \beth_{0+}} \operatorname{cod}^{*} \mathscr{H}_{i}(\bar{A}) \bigcup_{i \in \beth_{0+}}-\operatorname{cod}^{*} \mathscr{H}_{i}(\bar{A})\right. \\
& \left.\bigcup_{i \in \beth_{0}-\cup \beth_{00}}-\operatorname{cod}^{*} \mathscr{H}_{i}(\bar{A}) \bigcup_{i \in I_{+0}} \operatorname{cod}^{*} \mathscr{G}_{i}(\bar{A})\right)^{s} \bigcap M_{+}^{n}=\varnothing, \forall k \in I_{\mathfrak{f}} .
\end{aligned}
$$

Hence, GCCQ not satisfied.

Applying the generalized Guignard constraint qualification, we derive the KarushKuhn-Tucker type necessary optimality conditions for $(S-M M P V C)$.

Theorem 3. Suppose $\bar{A}$ is a local weak efficient solution for $(S-M M P V C)$. Assume that $\mathfrak{f}_{i}, \mathscr{H}_{i}, \mathscr{G}_{i}$ admits bounded upper semi-regular convexificator $\partial^{*} f_{i}(\bar{A})\left(i \in I_{\mathfrak{f}}\right), \partial^{*} \mathscr{H}_{i}(\bar{A})$ $\left(i \in I_{0}\right), \partial^{*} \mathscr{G}_{i}(\bar{A})\left(i \in I_{+0}\right)$, respectively, at $\bar{A}$. If (GGCQ) holds at $\bar{A}$ then there exists $\bar{\lambda}_{i}^{\mathfrak{f}}>0$ $\left(i \in \mathbb{I}_{\mathfrak{f}}\right), \bar{\lambda}^{\mathscr{G}} \in \mathbb{R}^{m}, \bar{\lambda}^{\mathscr{H}} \in \mathbb{R}^{m}$ and $\overline{\mathscr{W}} \in \mathbb{M}_{+}^{n}$ such that

$$
\begin{array}{r}
0 \in \sum_{i \in I_{\mathfrak{f}}} \bar{\lambda}_{i}^{\mathfrak{f}} \operatorname{cod}^{*} \mathfrak{f}_{i}(\bar{A})+\sum_{i=1}^{m}\left[\bar{\lambda}_{i}^{\mathscr{G}} \operatorname{cod}^{*} \mathscr{G}_{i}(\bar{A})-\bar{\lambda}_{i}^{\mathscr{H}} \operatorname{cod}^{*} \mathscr{H}_{i}(\bar{A})\right]-\overline{\mathscr{W}}, \\
\langle\overline{\mathscr{W}}, \bar{A}\rangle=0, \bar{\lambda}_{i}^{\mathscr{H}}=0\left(i \in \beth_{+0} \cup \beth_{+-}\right), \bar{\lambda}_{i}^{\mathscr{H}} \geqq 0\left(i \in \beth_{0-} \cup \beth_{00}\right), \bar{\lambda}_{i}^{\mathscr{H}} \text { free }\left(i \in \beth_{0+}\right), \\
\bar{\lambda}_{i}^{\mathscr{G}}=0\left(i \in \beth_{0+} \cup \beth_{0-} \cup \beth_{00} \cup \beth_{+-}\right), \bar{\lambda}_{i}^{\mathscr{G}} \geqq 0\left(i \in \beth_{+0}\right) .
\end{array}
$$

Proof. For the claim of the theorem, it suffices to show that,

$$
0 \in \sum_{i=1}^{p} \lambda_{i}^{\mathfrak{f}} \operatorname{co\partial }^{*} \mathfrak{f}_{i}(\bar{A})+C, \lambda^{\mathfrak{f}}>0 .
$$

Suppose, on the contrary, assume that

$$
0 \notin \sum_{i=1}^{p} \lambda_{i}^{\mathfrak{f}} \operatorname{co\partial }^{*} \mathfrak{f}_{i}(\bar{A})+C, \lambda^{\mathfrak{f}}>0 .
$$

As $\mathfrak{f}_{i}\left(i \in \beth_{\mathfrak{f}}\right)$ admits an upper semi-regular convexificator, this implies that the right side in (14) is a closed convex set in $\mathbb{M}^{n}$. The classical separation theorem implies that there exists $A \in \mathbb{M}^{n}$, such that

$$
\langle\tau, A\rangle<0, \forall \tau \in \sum_{i=1}^{p} \lambda_{i}^{\mathfrak{f}} \operatorname{cod}^{*} \mathfrak{f}_{i}(\bar{A})+C, \lambda^{\mathfrak{f}}>0 .
$$

Consequently,

$$
\left\langle\xi_{i}, A\right\rangle<0, \forall \xi_{i} \in \operatorname{cod}^{*} \mathfrak{f}_{i}(\bar{A})\left(i \in \beth_{\mathfrak{f}}\right),
$$




$$
\begin{gathered}
-\left\langle\eta_{i}, A\right\rangle \leqq 0, \forall \eta_{i} \in \operatorname{cod}^{*} \mathscr{H}_{i}(\bar{A})\left(i \in \beth_{0-} \cup I_{00}\right), \\
-\left\langle\eta_{i}, A\right\rangle \leqq 0, \forall \eta_{i} \in \operatorname{cod}^{*} \mathscr{H}_{i}(\bar{A})\left(i \in \beth_{0+}\right), \\
\left\langle\eta_{i}, A\right\rangle \leqq 0, \forall \eta_{i} \in \operatorname{cod}^{*} \mathscr{H}_{i}(\bar{A})\left(i \in \beth_{0+}\right), \\
\left\langle\zeta_{i}, A\right\rangle \leqq 0, \forall \zeta_{i} \in \operatorname{cod}^{*} \mathscr{G}_{i}(\bar{A})\left(i \in \beth_{+0}\right), \\
-\langle\overline{\mathscr{W}}, A\rangle \leqq 0, \forall \overline{\mathscr{W}} \in \mathbb{M}_{+}^{n} .
\end{gathered}
$$

Inequalities (17)-(22) and (GGCQ) implies that

$$
\begin{aligned}
& A \in\left(\bigcup_{i \in \Xi_{\mathfrak{f}}} \cot ^{*} \mathfrak{f}_{i}(\bar{A})\right)^{-} \bigcap\left(\bigcup_{i \in I_{0+}} \cot ^{*} \mathscr{H}_{i}(\bar{A}) \bigcup_{i \in I_{0+}}-\cot ^{*} \mathscr{H} \mathscr{H}_{i}(\bar{A})\right. \\
& \left.\bigcup_{i \in I_{0} \cup \beth_{00}}-\operatorname{cod}^{*} \mathscr{H}(\bar{A}) \bigcup_{i \in I_{+0}} \operatorname{cod}^{*} \mathscr{G}_{i}(\bar{A})\right)^{-} \bigcap M_{+}^{n} \subset \bigcap_{i=1}^{p} \operatorname{coT}\left(Q^{i}, \bar{A}\right) .
\end{aligned}
$$

Hence, $A \in \bigcap_{i=1}^{p} \operatorname{coT}\left(Q^{i}, \bar{A}\right)$, which implies that, there exist $t_{n} \downarrow 0$, such that $\bar{A}+t_{n} A \in$ $M$. Therefore, from (17), we obtain

$$
\mathfrak{f}_{i}(\bar{A}+t A)<\mathfrak{f}_{i}(\bar{A}), \forall i \in \mathfrak{I}_{\mathfrak{f}} .
$$

Thus, we obtain the contradiction that the feasible point $\bar{A}$ is a local weak efficient solution for $(S-M M P V C)$. Hence, the result.

Motivated by Achtziger and Kanzow [12] and Sadeghieh et al. [55], we define Sstationary point for S-MMPVC.

Definition 8. A feasible point $\bar{A}$ is said to be weak $S$-stationary point for $(S-M M P V C)$ if there exist $\lambda^{f} \in \mathbb{R}^{p}, \lambda^{\mathscr{H}} \in \mathbb{R}^{m}, \lambda^{\mathscr{G}} \in \mathbb{R}^{M}, \mathscr{W} \in \mathbb{M}_{+}^{n}$, and not all multipliers along with $\overline{\mathscr{W}}$ can be simultaneously zero, such that

$$
\begin{array}{r}
0 \in \sum_{i \in \beth_{\mathfrak{f}}} \lambda_{i}^{\mathfrak{f}} \operatorname{cod}^{*} \mathfrak{f}_{i}(\bar{A})+\sum_{i=1}^{m}\left[\lambda_{i}^{\mathscr{G}} \operatorname{cod}^{*} \mathscr{G}_{i}(\bar{A})-\lambda_{i}^{\mathscr{H}} \operatorname{cod}^{*} \mathscr{H}_{i}(\bar{A})\right]-\mathscr{W}, \\
\lambda_{i}^{\mathfrak{f}} \geqq 0\left(i \in \beth_{\mathfrak{f}}\right),\langle\mathscr{W}, \bar{A}\rangle=0, \lambda_{i}^{\mathscr{H}}=0\left(i \in \beth_{+0} \cup \beth_{+-}\right), \lambda_{i}^{\mathscr{H}} \geqq 0\left(i \in \beth_{0-} \cup \beth_{00}\right), \\
\lambda_{i}^{\mathscr{H}} \text { free }\left(i \in \beth_{0+}\right), \lambda_{i}^{\mathscr{G}}=0\left(i \in \beth_{0+} \cup \beth_{0-} \cup \beth_{00} \cup \beth_{+-}\right), \lambda_{i}^{\mathscr{G}} \geqq 0\left(i \in \beth_{+0}\right) .
\end{array}
$$

Definition 9. A feasible point $\bar{A}$ is said to be strong $S-$ stationary point for $(S-M M P V C)$ if there exist $\lambda^{\mathfrak{f}} \in \mathbb{R}^{p}, \lambda^{\mathscr{H}} \in \mathbb{R}^{m}, \lambda^{\mathscr{G}} \in \mathbb{R}^{M}$ and $\mathscr{W} \in \mathbb{M}_{+}^{n}$, such that

$$
\begin{aligned}
& 0 \in \sum_{i \in \beth_{\mathfrak{f}}} \lambda_{i}^{\mathfrak{f}} \operatorname{cod}^{*} \mathfrak{f}_{i}(\bar{A})+\sum_{i=1}^{m}\left[\lambda_{i}^{\mathscr{G}} \cot ^{*} \mathscr{G}_{i}(\bar{A})-\lambda_{i}^{\mathscr{H}} \cot ^{*} \mathscr{H}_{i}(\bar{A})\right]-\mathscr{W}, \\
& \lambda_{i}^{\mathfrak{f}}>0\left(i \in I_{\mathfrak{f}}\right),\langle\mathscr{W}, \bar{A}\rangle=0, \lambda_{i}^{\mathscr{H}}=0\left(i \in I_{+0} \cup I_{+-}\right), \lambda_{i}^{\mathscr{H}} \geqq 0\left(i \in I_{0-} \cup \beth_{00}\right) \text {, } \\
& \lambda_{i}^{\mathscr{H}} \text { free }\left(i \in \beth_{0+}\right), \lambda_{i}^{\mathscr{G}}=0\left(i \in \beth_{0+} \cup \beth_{0-} \cup \beth_{00} \cup \beth_{+-}\right), \lambda_{i}^{\mathscr{G}} \geqq 0\left(i \in I_{+0}\right) \text {. }
\end{aligned}
$$

Note that, if multipliers of gradients of objective functions are strictly greater than zero, then it is considered as strong $S$-stationary conditions. 
Example 2. Consider following optimization problem

$$
\begin{array}{r}
\min \left(\mathfrak{f}_{1}(A), \mathfrak{f}_{2}(A)\right) \text {, subject to } \mathscr{H}(A)=x_{1} \geqq 0, \mathscr{G}(A) \mathscr{H}(A)=x_{3} . x_{1} \leqq 0, \\
A=\left[\begin{array}{ll}
x_{1} & x_{2} \\
x_{2} & x_{3}
\end{array}\right] \in \mathbb{M}_{+}^{2} \text {, where } \mathfrak{f}_{1}(A)=\left|x_{1}-1\right|, \mathfrak{f}_{2}(A)=\left|x_{3}\right| .
\end{array}
$$

Feasible set $M=\left\{\left[\begin{array}{ll}x_{1} & x_{2} \\ x_{2} & x_{3}\end{array}\right] \in \mathbb{M}_{+}^{2}: x_{1} \geqq 0, x_{1} x_{3} \leqq 0\right\}$. Since $\bar{A}=\left[\begin{array}{ll}1 & 0 \\ 0 & 0\end{array}\right]$ is weak efficient solution for the considered problem. Now, we can find upper semi-regular convexificator of each functions at point $\bar{A}$ as follows:

$$
\begin{gathered}
\partial^{*} \mathfrak{f}_{1}(\bar{A})=\left\{\left[\begin{array}{cc}
-1 & 0 \\
0 & 0
\end{array}\right],\left[\begin{array}{ll}
1 & 0 \\
0 & 0
\end{array}\right]\right\}, \partial^{*} \mathfrak{f}_{2}(\bar{A})=\left\{\left[\begin{array}{cc}
0 & 0 \\
0 & -1
\end{array}\right],\left[\begin{array}{ll}
0 & 0 \\
0 & 1
\end{array}\right]\right\}, \\
\partial^{*} \mathscr{H}(\bar{A})=\left\{\left[\begin{array}{ll}
1 & 0 \\
0 & 0
\end{array}\right]\right\}, \partial^{*} \mathscr{G}(\bar{A})=\left\{\left[\begin{array}{ll}
0 & 0 \\
0 & 1
\end{array}\right]\right\} . \\
Q^{1}=\left\{\left[\begin{array}{ll}
x_{1} & x_{2} \\
x_{2} & x_{3}
\end{array}\right] \in \mathbb{M}_{+}^{2}: x_{1} \geqq 0, x_{2}=0, x_{3}=0\right\}, Q^{2}=\left\{\left[\begin{array}{ll}
x_{1} & x_{2} \\
x_{2} & x_{3}
\end{array}\right] \in \mathbb{M}_{+}^{2}: x_{1}=1, x_{2}=0, x_{3}=0\right\} .
\end{gathered}
$$

So, we conclude that

$$
\left[\begin{array}{ll}
0 & 0 \\
0 & 0
\end{array}\right] \in \bigcap_{i=1}^{2} \operatorname{coT}\left(Q^{i}, \bar{A}\right) \text { and } \bigcup_{i=1}^{2} \operatorname{coz}^{*} \mathfrak{f}_{i}(\bar{A})=\left\{\left[\begin{array}{ll}
t & 0 \\
0 & 0
\end{array}\right],\left[\begin{array}{ll}
0 & 0 \\
0 & s
\end{array}\right]: t, s \in[-1,1]\right\},
$$

thus, we have

$$
\left(\bigcup_{i=1}^{2} \operatorname{cod}^{*} f_{i}(\bar{A})\right)^{-}=\left\{\left[\begin{array}{ll}
x_{1} & x_{2} \\
x_{2} & x_{3}
\end{array}\right]: x_{1}=0, x_{2}=0, x_{3}=0\right\}
$$

Since,

$$
\operatorname{cod}^{*} \mathscr{H}(\bar{A})=\left\{\left[\begin{array}{ll}
1 & 0 \\
0 & 0
\end{array}\right]\right\} \text {, then }\left(-\cot ^{*} \mathscr{H}(\bar{A})\right)^{-}=\left\{\left[\begin{array}{ll}
x_{1} & x_{2} \\
x_{2} & x_{3}
\end{array}\right]: x_{1} \geqq 0\right\} .
$$

Consequently, we have

$$
\left(\bigcup_{i=1}^{2} \operatorname{coz}^{*} \mathfrak{f}_{i}(\bar{A})\right)^{-} \bigcap\left(-\operatorname{cod}^{*} \mathscr{H}(\bar{A})\right)^{-} \bigcap \mathbb{M}_{+}^{2}=\left\{\left[\begin{array}{ll}
0 & 0 \\
0 & 0
\end{array}\right]\right\} \subset \bigcap_{i=1}^{2} \cot \left(Q^{i}, \bar{A}\right) .
$$

Obviously, $C=$ cone $\operatorname{coz}^{*} \mathscr{H}(\bar{A})-\mathbb{M}_{+}^{2}$ is closed set. Hence, (GGCQ) satisfied at $\bar{A}$.

Now, for $\lambda_{1}^{\mathfrak{f}}=1, \lambda_{2}^{\mathfrak{f}}=1, \lambda^{\mathscr{H}}=0, \overline{\mathscr{W}}=\left[\begin{array}{ll}0 & 0 \\ 0 & 1\end{array}\right], \xi_{1}=\left[\begin{array}{ll}0 & 0 \\ 0 & 0\end{array}\right] \in \operatorname{cod}^{*} \mathfrak{f}_{1}(\bar{A})$, $\xi_{2}=\left[\begin{array}{ll}0 & 0 \\ 0 & 1\end{array}\right] \in \operatorname{cod}^{*} \mathfrak{f}_{2}(\bar{A})$, and $\eta=\left[\begin{array}{ll}1 & 0 \\ 0 & 0\end{array}\right] \in \operatorname{cod}^{*} \mathscr{H}(\bar{A})$, we have

$$
\begin{aligned}
& 0=\lambda_{1}^{\mathfrak{f}} \xi_{1}+\lambda_{2}^{\mathfrak{f}} \xi_{2}-\lambda^{\mathscr{H}} \eta-\overline{\mathscr{W}}=1\left[\begin{array}{ll}
0 & 0 \\
0 & 0
\end{array}\right]+1\left[\begin{array}{ll}
0 & 0 \\
0 & 1
\end{array}\right]-0\left[\begin{array}{ll}
1 & 0 \\
0 & 0
\end{array}\right]-\left[\begin{array}{ll}
0 & 0 \\
0 & 1
\end{array}\right] \\
& \in \lambda_{1}^{\mathfrak{f}} \operatorname{coz}^{*} \mathfrak{f}_{1}(\bar{A})+\lambda_{2}^{\mathfrak{f}} \operatorname{co} \partial^{*} \mathfrak{f}_{2}(\bar{A})-\lambda^{\mathscr{H}} \operatorname{co\partial }{ }^{*} \mathscr{H}(\bar{A})-\overline{\mathscr{W}},
\end{aligned}
$$

and $\langle\bar{A}, \overline{\mathscr{W}}\rangle=\operatorname{Tr}\left(\left[\begin{array}{ll}1 & 0 \\ 0 & 0\end{array}\right]\left[\begin{array}{ll}0 & 0 \\ 0 & 1\end{array}\right]\right)=0$. Hence, strong $S$-stationary conditions satisfied at weak efficient point $\bar{A}$. 
Corollary 1. Let $\bar{A}$ be a local weak efficient solution for $(S-M M P V C)$. Suppose that $\mathfrak{f}_{i}, \mathscr{H}_{i}, \mathscr{G}_{i}$ admits bounded upper semi-regular convexificator $\partial^{*} \mathfrak{f}_{i}(\bar{A})\left(i \in \beth_{\mathfrak{f}}\right), \partial^{*} \mathscr{H}_{i}(\bar{A})\left(i \in \beth_{0}\right), \partial^{*} \mathscr{G}_{i}(\bar{A})$ $\left(i \in \beth_{+0}\right)$, respectively, at $\bar{A}$. If (GGCQ) holds at $\bar{A}$ then there exists $\bar{\lambda}_{i}^{\mathfrak{f}}>0\left(i \in I_{\mathfrak{f}}\right)$, $\bar{\lambda}^{\mathscr{G}} \in \mathbb{R}^{m}, \bar{\lambda}^{\mathscr{H}} \in \mathbb{R}^{m}$ and $\overline{\mathscr{W}} \in \mathbb{M}_{+}^{n}$ such that

$$
\begin{array}{r}
0 \in \sum_{i \in I_{\mathfrak{f}}} \bar{\lambda}_{i}^{\mathfrak{f}} \operatorname{cod}^{*} \mathfrak{f}_{i}(\bar{A})+\sum_{i=1}^{m}\left[\bar{\lambda}_{i}^{\mathscr{G}} \operatorname{cod}^{*} \mathscr{G}_{i}(\bar{A})-\bar{\lambda}_{i}^{\mathscr{H}} \operatorname{co\partial }{ }^{*} \mathscr{H}_{i}(\bar{A})\right]-\overline{\mathscr{W}}, \\
\langle\overline{\mathscr{W}}, \bar{A}\rangle=0, \bar{\lambda}_{i}^{\mathscr{H}}=0\left(i \in \beth_{+0} \cup \beth_{+-}\right), \bar{\lambda}_{i}^{\mathscr{H}} \geqq 0\left(i \in \beth_{0-} \cup \beth_{00}\right), \bar{\lambda}_{i}^{\mathscr{H}} \text { free }\left(i \in \beth_{0+}\right), \\
\sum_{i=1}^{p} \bar{\lambda}_{i}^{\mathfrak{f}}=1, \bar{\lambda}_{i}^{\mathscr{G}}=0\left(i \in \beth_{0+} \cup \beth_{0-} \cup \beth_{00} \cup \beth_{+-}\right), \bar{\lambda}_{i}^{\mathscr{G}} \geqq 0\left(i \in I_{+0}\right) .
\end{array}
$$

Proof. Since, all conditions of Theorem 3 are satisfying for some $\lambda^{\mathfrak{f}}>0, \lambda^{\mathscr{H}}, \lambda^{\mathscr{G}} \in \mathbb{R}^{m}$, and $\mathscr{W}$ as follows:

$$
\begin{array}{r}
0 \in \sum_{i \in I_{\mathfrak{f}}} \lambda_{i}^{\mathfrak{f}} \operatorname{cod}^{*} \mathfrak{f}_{i}(\bar{A})+\sum_{i=1}^{m}\left[\lambda_{i}^{\mathscr{G}} \operatorname{co} \partial^{*} \mathscr{G}_{i}(\bar{A})-\lambda_{i}^{\mathscr{H}} \operatorname{co} \partial^{*} \mathscr{H}_{i}(\bar{A})\right]-\mathscr{W}, \\
\langle\mathscr{W}, \bar{A}\rangle=0, \lambda_{i}^{\mathscr{H}}=0\left(i \in \beth_{+0} \cup \beth_{+-}\right), \lambda_{i}^{\mathscr{H}} \geqq 0\left(i \in \beth_{0-} \cup \beth_{00}\right), \\
\lambda_{i}^{\mathscr{H}} \text { free }\left(i \in \beth_{0+}\right), \lambda_{i}^{\mathscr{G}}=0\left(i \in \beth_{0+} \cup \beth_{0-} \cup \beth_{00} \cup \beth_{+-}\right), \lambda_{i}^{\mathscr{G}} \geqq 0\left(i \in \beth_{+0}\right) .
\end{array}
$$

Now, dividing (23) by $\sum_{i=1}^{p} \lambda_{i}^{\mathfrak{f}}$ and taking

$$
\bar{\lambda}_{i}^{\mathfrak{f}}=\frac{\lambda_{i}^{\mathfrak{f}}}{\sum_{i=1}^{p} \lambda_{i}}, \bar{\lambda}_{i}^{\mathscr{H}}=\frac{\lambda_{i}^{\mathscr{H}}}{\sum_{i=1}^{p} \lambda_{i}^{\mathfrak{f}}}, \bar{\lambda}_{i}^{\mathscr{G}}=\frac{\lambda_{i}^{\mathscr{G}}}{\sum_{i=1}^{p} \lambda_{i}^{\mathfrak{f}}}, \overline{\mathscr{W}}=\frac{\mathscr{W}}{\sum_{i=1}^{p} \lambda_{i}^{\mathfrak{f}}},
$$

we obtain the required result.

Now, we propose some index sets to show sufficient optimality conditions for SMMPVC:

$$
\begin{array}{r}
\beth_{00}^{+}:=\left\{i \in \beth_{00}: \lambda_{i}^{\mathscr{H}}>0\right\}, \\
\beth_{00}^{0}:=\left\{i \in \beth_{00}: \lambda_{i}^{\mathscr{H}}=0\right\}, \\
\beth_{0-}^{+}:=\left\{i \in \beth_{0-}: \lambda_{i}^{\mathscr{H}}>0\right\}, \\
\beth_{0-}^{0}:=\left\{i \in \beth_{0-}: \lambda_{i}^{\mathscr{H}}=0\right\}, \\
\beth_{0+}^{+}:=\left\{i \in \beth_{0+}: \lambda_{i}^{\mathscr{H}}>0\right\}, \\
\beth_{0+}^{-}:=\left\{i \in \beth_{0+}: \lambda_{i}^{\mathscr{H}}<0\right\}, \\
\beth_{0+}^{0}:=\left\{i \in \beth_{0+}: \lambda_{i}^{\mathscr{H}}=0\right\}, \\
\beth_{+0}^{0+}:=\left\{i \in \beth_{+0}: \lambda_{i}^{\mathscr{H}}=0, \lambda_{i}^{\mathscr{G}}>0\right\}, \\
\beth_{+0}^{00}:=\left\{i \in \beth_{+0}: \lambda_{i}^{\mathscr{H}}=0, \lambda_{i}^{\mathscr{G}}=0\right\} .
\end{array}
$$

Following result is motivated by Sadeghieh et al. ([55], Theorem 9).

Theorem 4. (Sufficient conditions) Suppose $\mathfrak{f}_{i}\left(i \in \beth_{\mathfrak{f}}\right), \mathscr{H}_{i}\left(i \in \beth_{0+} \cup \beth_{00} \cup \beth_{0-}\right), \mathscr{G}_{i}\left(i \in \beth_{+0}\right)$ admit bounded upper semi-regular convexificators at $\bar{A}$. Assume that feasible point $\bar{A}$ satisfies weak $S$-stationary conditions under suitable choice of multipliers $\lambda^{\mathfrak{f}} \in \mathbb{R}^{p}, \lambda^{\mathscr{H}} \in \mathbb{R}^{m}$, $\lambda^{\mathscr{G}} \in \mathbb{R}^{m}, \overline{\mathscr{W}} \in \mathbb{M}_{+}^{n}$ for $S-M M P V C$. If $\mathscr{H}_{i}\left(i \in I_{0+}^{-}\right),-\mathscr{H}_{i}\left(i \in I_{0+}^{+} \cup \beth_{00}^{+} \cup \beth_{0-}^{+}\right)$, $\mathscr{G}_{i}\left(i \in \beth_{+0}^{0+}\right)$, are $\partial^{*}-$ quasiconvex and $\mathfrak{f}_{i}\left(i \in I_{\mathfrak{f}}\right)$ are $\partial^{*}$-pseudoconvex at $\bar{A}$ and at least one $\lambda_{i}^{\mathfrak{f}}>0$. Then,

(i) $\bar{A}$ is a local weak efficient solution for $S-M M P V C$; 
(ii) In addition to that if $\beth_{0+}^{-} \cup \beth_{+0}^{0+}=\varnothing$, then $\bar{A}$ is a weak efficient solution for $S-M M P V C$.

Proof. (i) From continuity of $\mathscr{G}_{i}\left(i \in \beth_{0+}\right)$ and $\mathscr{H}_{i}\left(i \in \beth_{+0}\right)$ there exist neighborhoods $\mathcal{N}$ and $\mathcal{M}$ for $\bar{A}$, such that

$$
\begin{aligned}
& \mathscr{H}_{i}(A)=0, \mathscr{G}_{i}(A)>0, \forall A \in M \cap \mathcal{N} \forall i \in \beth_{0+}, \\
& \mathscr{H}_{i}(A)>0, \mathscr{G}_{i}(A) \leq 0, \forall A \in M \cap \mathcal{M} \forall i \in \beth_{+0} .
\end{aligned}
$$

Since $\bar{A}$ is a weak $S$-stationary point, so there exist $\lambda^{\mathfrak{f}} \in \mathbb{R}^{p}, \lambda^{\mathscr{H}} \in \mathbb{R}^{m}, \lambda^{\mathscr{G}} \in \mathbb{R}^{m}, \overline{\mathscr{W}}$ and not all multipliers along with $\overline{\mathscr{W}}$ can be simultaneously zero, such that satisfies weak $S-$ stationary conditions. Thus, there exist $\xi_{i} \in \operatorname{cod}^{*} \mathfrak{f}_{i}(\bar{A})\left(i \in I_{\mathfrak{f}}\right), \eta_{i} \in \operatorname{cod}^{*} \mathscr{H}_{i}(\bar{A})$ $\left(i \in \beth_{0}\right), \zeta_{i} \in \operatorname{cod}^{*} \mathscr{G}_{i}(\bar{A})\left(i \in \beth_{+0}\right)$, such that

$$
\sum_{i \in I_{\mathfrak{f}}} \lambda_{i}^{\mathfrak{f}} \xi_{i}+\sum_{i \in \beth_{+0}} \lambda_{i}^{\mathscr{G}} \zeta_{i}-\sum_{i \in \beth_{0}} \lambda_{i}^{\mathscr{H}} \eta_{i}-\overline{\mathscr{W}}=0 .
$$

Suppose, on contrary $\bar{A}$ is not local weak efficient solution for $S-M M P V C$. Then, there exists $B \in M \cap \mathcal{N} \cap \mathcal{M}$, such that

$$
\mathfrak{f}_{i}(B)<\mathfrak{f}_{i}(\bar{A}), \forall i \in \beth_{\mathfrak{f}}
$$

By the $\partial^{*}$-pseudoconvexity of $\mathfrak{f}_{i}\left(i \in I_{\mathfrak{f}}\right)$ and (27), we obtain

$$
\left\langle\xi_{i}, B-\bar{A}\right\rangle<0, \forall i \in \beth_{\mathfrak{f}} .
$$

By the $\partial^{*}$-quasiconvexity of functions $\mathscr{G}_{i}\left(i \in \beth_{+0}^{0+}\right), \mathscr{H}_{i}\left(i \in \beth_{0+}^{-}\right)$and (24) and (25), we obtain

$$
\begin{aligned}
& \mathscr{G}_{i}(B) \leqq 0=\mathscr{G}_{i}(\bar{A}) \Longrightarrow\left\langle\zeta_{i}, B-\bar{A}\right\rangle \leqq 0, \forall i \in \beth_{+0}^{0+} . \\
& \mathscr{H}_{i}(B)=0 \leqq \mathscr{H}_{i}(\bar{A}) \Longrightarrow\left\langle\eta_{i}, B-\bar{A}\right\rangle \leqq 0, \forall i \in \beth_{0+}^{-} .
\end{aligned}
$$

On the other hand, $\forall i \in \beth_{0+}^{+} \cup \beth_{0-}^{+} \cup \beth_{00^{\prime}}^{+}$

$$
-\mathscr{H}_{i}(B) \leqq 0=-\mathscr{H}_{i}(\bar{A}) \Longrightarrow\left\langle-\eta_{i}, B-\bar{A}\right\rangle \leqq 0, \forall-\eta_{i} \in-\operatorname{coz}^{*} \mathscr{H}_{i}(\bar{A}) \text {. }
$$

Since $\overline{\mathscr{W}}, B \in \mathbb{M}_{+}^{n}$, so we have

$$
-\langle\overline{\mathscr{W}}, B\rangle+\langle\overline{\mathscr{W}}, \bar{A}\rangle=-\langle\overline{\mathscr{W}}, B-\bar{A}\rangle \leqq 0
$$

Multiplying their corresponding multiplier in (29) to (32) and adding, we obtain contradictions to (26). Hence, the result.

(ii) We proceed similar to $(i)$ and using $\beth_{0+}^{+0} \cup \beth_{+0}^{-}=\varnothing$, therefore without making use of neighborhood $\mathcal{N}$ and $\mathcal{M}$, we obtain the required result.

To validate the sufficient optimality conditions we present following example.

Example 3. Consider following optimization problem

$\min \left(\mathfrak{f}_{1}(A), \mathfrak{f}_{2}(A)\right)$, subject to $\mathscr{H}_{1}(A)=-x_{2} \geqq 0, \mathscr{G}_{1}(A) \mathscr{H}_{1}(A)=-\left|x_{3}\right| x_{2} \leqq 0$,

$$
A=\left[\begin{array}{ll}
x_{1} & x_{2} \\
x_{2} & x_{3}
\end{array}\right] \in \mathbb{M}_{+}^{2} \text {, where } \mathfrak{f}_{1}(A)=x_{2}, \mathfrak{f}_{2}(A)=x_{3} \text {. }
$$


Feasible set,

$$
\begin{aligned}
M & =\left\{\left[\begin{array}{ll}
x_{1} & x_{2} \\
x_{2} & x_{3}
\end{array}\right] \in \mathbb{M}_{+}^{2}: x_{2} \leqq 0,\left|x_{3}\right| x_{2} \geqq 0\right\}, \\
& =\left\{\left[\begin{array}{ll}
x_{1} & x_{2} \\
x_{2} & x_{3}
\end{array}\right]: x_{1} \geqq 0, x_{1} x_{3}-x_{2}^{2} \geqq 0, x_{2} \leqq 0,\left|x_{3}\right| x_{2} \geqq 0\right\} .
\end{aligned}
$$

Consider at feasible point $\bar{A}=\left[\begin{array}{ll}0 & 0 \\ 0 & 0\end{array}\right]$. We observe that $\mathfrak{f}_{1}, \mathfrak{f}_{2}$ are $\partial^{*}-$ pseudoconvex, $-\mathscr{H}_{1}$ is $\partial^{*}$-quasiconvex at $\bar{A}$ and $\mathscr{H}_{i}\left(i=1 \in \beth_{00}\right), \mathscr{G}_{i}\left(i=1 \notin \beth_{+0}\right)$ also $\beth_{0+}^{+0} \cup \beth_{+0}^{-}=\varnothing$. Now, we can find upper semi-regular convexificator of each functions at point $\bar{A}$ as follows:

$$
\partial^{*} \mathfrak{f}_{1}(\bar{A})=\left\{\left[\begin{array}{ll}
0 & \frac{1}{2} \\
\frac{1}{2} & 0
\end{array}\right]\right\}, \partial^{*} \mathfrak{f}_{2}(\bar{A})=\left\{\left[\begin{array}{ll}
0 & 0 \\
0 & 1
\end{array}\right]\right\}, \partial^{*} \mathscr{H}_{1}(\bar{A})=\left\{\left[\begin{array}{cc}
0 & -\frac{1}{2} \\
-\frac{1}{2} & 0
\end{array}\right]\right\} .
$$

Thus, for $\lambda_{1}^{\mathfrak{f}}=0, \lambda_{2}^{\mathfrak{f}}>0, \lambda_{1}^{\mathscr{H}}=0$, and $\overline{\mathscr{W}}=\left[\begin{array}{cc}0 & 0 \\ 0 & \lambda_{2}^{\mathfrak{f}}\end{array}\right]$, we have

$$
\lambda_{1}^{\mathfrak{f}} \operatorname{cod}^{*} \mathfrak{f}_{1}(\bar{A})+\lambda_{2}^{\mathfrak{f}} \operatorname{cod}^{*} \mathfrak{f}_{2}(\bar{A})-\lambda_{1}^{\mathscr{H}} \operatorname{cod}^{*} \mathscr{H}_{1}(\bar{A})-\overline{\mathscr{W}}=0 .
$$

That is, $\bar{A}$ satisfying weak S-stationary conditions. Hence, $\bar{A}$ is weak efficient solution, which is true by simple observations.

\section{Conclusions and Future Remarks}

Golestani and Nobakhtian [11] established optimality conditions for nonsmooth semidefinite single optimization problems. We have established the optimality conditions for a more interesting class of nonlinear optimization namely, mathematical programming problems with vanishing constraints (MPVC), which is more applicable in topology optimization and many real-life problems. We have further extended the single objective semidefinite optimization problems to multiobjective semidefinite optimization problems. We established Fritz John stationary conditions for nonsmooth, nonlinear, semidefinite, multiobjective programs with vanishing constraints using convexificator and generalized Cottle type and generalized Guignard type constraints qualification have been introduced to achieve strong $S$-stationary conditions from Fritz John stationary conditions. Sufficient conditions are also established under generalized convexity assumptions and through an example, we validate our established results. We have used the constraint qualifications technique motivated by $\mathrm{Li}$ [38] and provided some generalized constraint qualifications for semidefinite optimization problems. We have also used the linearization technique inspired by Kanzow et al. [56]. Recently, Treanta [41] discussed duality theorems for a special class of quasiinvex multiobjective optimization problems for interval-valued components. Further, Treanta established dual pair of multiobjective interval-valued variational control problems. We can extend the results on multiobjective semidefinite optimization problems to variational control problems and interval-valued optimization problems motivated by $[40,41,57-61]$ for the application point of view.

Author Contributions: Writing-original draft preparation, K.K.L., M.H., S.K.S., J.K.M. and S.K.M.; writing-review and editing, K.K.L., M.H., S.K.S., J.K.M. and S.K.M.; funding acquisition, K.K.L. All authors have read and agreed to the published version of the manuscript.

Funding: The second author is financially supported by CSIR-UGC JRF, New Delhi, India, through Reference no.: 1009/(CSIR-UGC NET JUNE 2018). The third author is financially supported by CSIR-UGC JRF, New Delhi, India, through Reference no.: 1272/(CSIR-UGC NET DEC.2016). The fifth author is financially supported by "Research Grant for Faculty" (IoE Scheme) under Dev. Scheme NO. 6031 and Department of Science and Technology, SERB, New Delhi, India, through grant no.: MTR/2018/000121. 
Institutional Review Board Statement: Not Applicable.

Informed Consent Statement: Not Applicable.

Data Availability Statement: No data were used to support this study.

Acknowledgments: The authors are indebted to the anonymous reviewers for their valuable comments and remarks that helped to improve the presentation and quality of the manuscript.

Conflicts of Interest: The authors declare no conflict of interest.

\section{References}

1. Lobo, M.S.; Vandenberghe, L.; Boyd, S.; Lebret, H. Applications of second-order cone programming. Linear Algebra Its Appl. 1998, 284, 193-228. [CrossRef]

2. Vandenberghe, L.; Boyd, S. Semidefinite programming. SIAM Rev. 1996, 38, 49-95. [CrossRef]

3. Fares, B.; Noll, D.; Apkarian, P. Robust control via sequential semidefinite programming. SIAM J. Control Optim. 2002, 4, 1791-1820. [CrossRef]

4. Ben-Tal, A.; Jarre, F.; Kočvara, M.; Nemirovski, A.; Zowe, J. Optimal design of trusses under a nonconvex global buckling constraint. Optim. Eng. 2000, 1, 189-213. [CrossRef]

5. Leibfritz, F. COMPleib 1.1: COnstraint Matrix-Optimization Problem Library-A Collection of Test Examples for Nonlinear Semidefinite Programs, Control System Design and Related Problems; Technical Report; Department of Mathematics, University of Trier: Trier, Germany, 2005.

6. Shapiro, A. First and second order analysis of nonlinear semidefinite programs. Math. Program. 1997, 77, 301-320. [CrossRef]

7. Sun, D.; Sun, J.; Zhang, L. The rate of convergence of the augmented Lagrangian method for nonlinear semidefinite programming. Math. Program. Ser. A 2008, 114, 349-391. [CrossRef]

8. Forsgren, A. Optimality conditions for nonconvex semidefinite programming. Math. Program. Ser. A 2000, 88, 105-128. [CrossRef]

9. Sun, J. On method for solving nonlinear semidefinite optimization problems. Numer. Algebr. Control Optim. 2011, 1, 1-14. [CrossRef]

10. Yamashita, H.; Yabe, H. A survey of numerical methods doe nonlinear semidefinite programming. J. Oper. Res. Soc. Jpn. 2015, 58, 24-60.

11. Golestani, M.; Nobakhtian, S. Optimality conditions for nonsmooth semidefinite programming via convexificators. Positivity 2015, 19, 221-236. doi:10.1007/s11117-014-0292-6. [CrossRef]

12. Achtziger, W.; Kanzow, C. Mathematical programs with vanishing constraints: optimality conditions and constraint qualifications. Math. Program. Ser. A 2008, 114, 69-99. [CrossRef]

13. Kirches, C.; Potschka, A.; Bock, H.G.; Sager, S. A parametric active-set method for QPS with vanishing constraints arising in a robot motion planning problem. Pac. J. Optim. 2013, 9, 275-299.

14. Jung, M.N.; Kirches, C.; Sager, S. On Perspective Functions and Vanishing Constraints in Mixed-Integer Nonlinear Optimal Control; Springer: Berlin/Heidelberg, Germany, 2013; pp. 387-417.

15. Jabr, R. Solution to economic dispatching with disjoint feasible regions via semidefinite programming. IEEE Trans. Power Syst. 2012, 27, 572-573. [CrossRef]

16. Guu, S.M.; Singh, Y.; Mishra, S.K. On strong KKT type sufficient optimality conditions for multiobjective semi-infinite programming problems with vanishing constraints. J. Inequalities Appl. 2017, 2017, 1-9. [CrossRef] [PubMed]

17. Mishra, S.K.; Singh, V.; Laha, V. On duality for mathematical programs with vanishing constraints. Ann. Oper. Res. 2016, 243, 249-272. [CrossRef]

18. Mishra, S.K.; Singh, V.; Laha, V.; Mohapatra, R.N. On constraint qualifications for multiobjective optimization problems with vanishing constraints. In Optimization Methods, Theory and Applications; Springer: Berlin/Heidelberg, Germany, 2015 ; pp. 95-135.

19. Luo, Z.Q.; Pang, J.S.; Ralph, D. Mathematical Programs with Equilibrium Constraints; Cambridge University Press: Cambridge, $\mathrm{UK}, 1996$.

20. Outrata, J.; Kocvara, M.; Zowe, J. Nonsmooth approach to optimization problems with equilibrium constraints. In Nonconvex Optimization and Its Applications; Kluwer Academic Publishers: Dordrecht, The Netherlands, 1998.

21. Mishra, S.K.; Jaiswal, M. Optimality conditions and duality for semi-infinite mathematical programming problem with equilibrium constraints. Numer. Funct. Anal. Optim. 2015, 36, 460-480. [CrossRef]

22. Pandey, Y.; Mishra, S.K. Optimality conditions and duality for semi-infinite mathematical programming problems with equilibrium constraints, using convexificators. Ann. Oper. Res. 2018, 269, 549-564. [CrossRef]

23. Pandey, Y.; Mishra, S.K. On strong KKT type sufficient optimality conditions for nonsmooth multiobjective semi-infinite mathematical programming problems with equilibrium constraints. Oper. Res. Lett. 2016, 44, 148-151. [CrossRef]

24. Singh, Y.; Pandey, Y.; Mishra, S.K. Saddle point optimality criteria for mathematical programming problems with equilibrium constraints. Oper. Res. Lett. 2017, 45, 254-258. [CrossRef]

25. Guu, S.M.; Mishra, S.K.; Pandey, Y. Duality for nonsmooth mathematical programming problems with equilibrium constraints. J. Inequalities Appl. 2016, 2016, 28. [CrossRef] 
26. Pandey, Y.; Mishra, S.K. Duality of mathematical programming problems with equilibrium constraints. Pac. J. Optim. 2017, 13, 105-122.

27. Mishra, S.K.; Pandey, Y. On sufficiency for mathematical programming problems with equilibrium constraints. Yugosl. J. Oper. Res. 2013, 23, 173-182.

28. Pandey, Y.; Mishra, S.K. Duality for nonsmooth optimization problems with equilibrium constraints, using convexificators. J. Optim. Theory Appl. 2016, 171, 694-707. [CrossRef]

29. Hoheisel, T.; Kanzow, C. First- and second-order optimality conditions for mathematical programs with vanishing constraints. Appl. Math. 2007, 52, 495-514. [CrossRef]

30. Hoheisel, T.; Kanzow, C. Stationary conditions for mathematical programs with vanishing constraints using weak constraint qualifications. J. Math. Anal. Appl. 2008, 337, 292-310. [CrossRef]

31. Hoheisel, T.; Kanzow, C. On the Abadie and Guignard constraint qualifications for mathematical programmes with vanishing constraints. Optimization 2009, 58, 431-448. [CrossRef]

32. Hoheisel, T.; Kanzow, C.; Outrata, J.V. Exact penalty results for mathematical programs with vanishing constraints. Nonlinear Anal 2010, 72, 2514-2526. [CrossRef]

33. Izmailov, A.F.; Pogosyan, A.L. Optimality conditions and newton-type methods for mathematical programs with vanishing constraints. Comput. Math. Math. Phys. 2009, 49, 1128-1140. [CrossRef]

34. Miettinen, K. Nonlinear Multiobjective Optimization; Kluwer Academic Publishers: Boston, MA, USA, 1999.

35. Wendell, R.E.; Lee, D.N. Efficiency in multiple objective optimization problems. Math. Program 1977, 12, 406-414. [CrossRef]

36. Maeda, T. Constraint qualifications in multiobjective optimization problems: Differentiable case. J. Optim. Theory Appl. 1994, 80, 483-500. [CrossRef]

37. Preda, V.; Chițescu, I. On constraint qualification in multiobjective optimization problems: Semidifferentiable case. J. Optim. Theory Appl. 1999, 100, 417-433. [CrossRef]

38. Li, X.F. Constraint qualifications in nonsmooth multiobjective optimization. J. Optim. Theory Appl. 2000, 106, 373-398. [CrossRef]

39. Lai, K.K.; Hassan, M.; Maurya, J.K.; Singh, S.K.; Mishra, S.K. Multiobjective Convex Optimization in Real Banach Space. Symmetry 2021, 13, 2148. [CrossRef]

40. Treanţă, S. On a Dual Pair of Multiobjective Interval-Valued Variational Control Problems. Mathematics 2021, 9, 893. [CrossRef]

41. Treanta, S. Duality Theorems for $(\rho, \psi, d)$-Quasiinvex multiobjective optimization problems with interval-valued components. Mathematics 2021, 9, 894. [CrossRef]

42. Demyanov, V.F.; Jeyakumar, V. Hunting for a smaller convex subdifferential. J. Glob. Optim. 1997, 10, 305-326. [CrossRef]

43. Jeyakumar, V.; Luc, D.T. Nonsmooth calculus, minimality, and monotonicity of convexificators. J. Optim. Theory Appl. 1999, 101, 599-621. [CrossRef]

44. Dorsch, D.; Gómez, W.; Shikhman, V. Sufficient optimality conditions hold for almost all nonlinear semidefinite programs. Math Program. 2016, 158, 77-97. [CrossRef]

45. Yurtsever, A.; Tropp, J.A.; Fercoq, O.; Udell, M.; Cevherk, V. Scalable Semidefinite Programming. SIAM J. Math. Data Sci. 2021, 3, 171-200. [CrossRef]

46. Andreani, R.; Haeser, G.; Viana, D.S. Optimality conditions and global convergence for nonlinear semidefinite programming Math. Program. Ser. A 2020, 180, 203-235. [CrossRef]

47. Andreani, R.; Haeser, G.; Mito, L.M.; Ramírez, H.; Santos, D.O.; Silveira, T.P. Naive constant rank-type constraint qualifications for multifold second-order cone programming and semidefinite programming. Optim. Lett. 2021. 2021, 1-22. doi: 10.1007/s11590-02101737-w. [CrossRef]

48. Berman, A.; Shaked-Monderer, N. Completely Positive Matrices; World Scientific Publishing Co., Inc.: River Edge, NJ, USA, 2003.

49. Boyd, S.; El Ghaoui, L.; Feron, E.; Balakrishnan, V. Linear Matrix Inequalities in System and Control Theory. In SIAM Studies in Applied Mathematics; Society for Industrial and Applied Mathematics (SIAM): Philadelphia, PA, USA, 1994 ; Volume 15.

50. Clarke, F.H. Optimization and Nonsmooth Analysis, Classics in Applied Mathematics, 2nd ed.; Society for Industrial and Applied Mathematics (SIAM): Philadelphia, PA, USA, 1990; Volume 5.

51. Michel, P.; Penot, J.P. A generalized derivative for calm and stable functions. Differ. Integr. Equ. 1992, 5, $433-454$.

52. Mordukhovich, B.S.; Shao, Y.H. On nonconvex subdifferential calculus in Banach spaces. J. Convex Anal. 1995, 2, $211-227$.

53. Treiman, J.S. The linear nonconvex generalized gradient and Lagrange multipliers. SIAM J. Optim. 1995, 5, 670-680. [CrossRef]

54. Fan, J. Generalized separation theorems and the Farkas' lemma. Appl. Math. Lett. 2005, 18, 791-796. [CrossRef]

55. Sadeghieh, A.; Kanzi, N.; Caristi, G.; Barilla, D. On stationarity for nonsmooth multiobjective problems with vanishing constraints. J. Glob. Optim. 2021., 2021, 1-21. doi: 10.1007/s10898-021-01030-1. [CrossRef]

56. Kanzow, C.; Nagel, C.; Kato, H.; Fukushima, M. Successive linearization methods for nonlinear semidefinite programs. Comput. Optim. Appl. 2005, 31, 251-273. [CrossRef]

57. Treanţă, S. On locally and globally optimal solutions in scalar variational control problems. Mathematics 2019, 7, 829. [CrossRef]

58. Treanta, S.; Udriste, C. On efficiency conditions for multiobjective variational problems involving higher order derivatives. In Proceedings of the 15th International Conference on Automatic Control, Modelling \& Simulation (ACMOS'13), Brasov, Romania, 1-3 June 2013; pp. 157-164.

59. Treanţă, S. Well Posedness of New Optimization Problems with Variational Inequality Constraints. Fractal Fract. 2021, 5, 123. [CrossRef] 
60. Mititelu, Ş.; Treanţă, S. Efficiency conditions in vector control problems governed by multiple integrals. J. Appl. Math. Comput. 2018, 57, 647-665. [CrossRef]

61. Treanţă, S. On modified interval-valued variational control problems with first-order PDE constraints. Symmetry 2020, $12,472$. [CrossRef] 